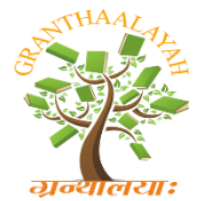

\author{
INTERNATIONAL JOURNAL OF RE
GRANTHAALAYAH \\ A knowledge Repository
}

Science

\title{
ASSESSMENT OF SURFACE WATER RESOURCES FOR IRRIGATION PURPOSES IN ASSIUT GOVERNORATE, UPPER EGYPT
}

\author{
Mamdouh Salama Morsi ${ }^{1}$, Abdel Hai. A. Farrag ${ }^{2}$, Esam E. A. El Sayed ${ }^{3}$, Ashraf M. T. \\ Elewa $^{3}$ \\ ${ }^{1}$ Mining Authority, Minia Governorate, Egypt \\ ${ }^{2}$ Geology Department, Faculty of Science, Assiut University, Egypt \\ ${ }^{3}$ Geology Department, Faculty of Science, Minia University, Egypt

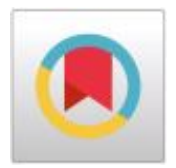

\begin{abstract}
Water from some sources may contain so much salt that it is unsuitable for irrigation because of potential danger to the soil or crops. Irrigation water quality can best be determined by chemical laboratory analysis. The main objectives of this study are to assess the surface water quality for irrigation, and to present solutions for managing and protecting these resources in Assiut area. To achieve that, thirty surface water samples were collected from River Nile and main irrigation canals. Chemical analysis was carried out and analyzed for major and trace elements according to the irrigation water guidelines of (FAO 1985), and (Rowe, et al. 1995), taking into account the spatial variations and the representation of the hydro chemical data. The results show that $97 \%$ of surface water samples lie within no restriction on use level and 3\% is represent slight to moderate restoration on use according to TDS concentrations. $97 \%$ of surface water samples belongs to (C2-S1) good water for irrigation all crops in all soils and $3 \%$ belongs to (C3 -S1) good water for irrigation all crops in all soils under ordinary and specific condition like adequate drainage and leaching According U.S. salinity laboratory staff classification depend on (EC, TDS and SAR). Where $87 \%$ Excellent water for irrigation sensitive all crops and low likelihood of soil problems According Boron content. Consequently, it is recommended to prevent the sewage and domestic waste water, and the industrial waste water from direct disposal without treatment to the irrigation canals and River Nile; controlling the use of fertilizers and pesticides in the agriculture purposes; selected the suitable crops for every sector (area) according to the chemical characters of the available irrigation water and soil properties.
\end{abstract}

Keywords: Surface Water; (FAO 1985); Irrigation; Assiut Area; River Nile; Egypt.

Cite This Article: Mamdouh Salama Morsi, Abdel Hai. A. Farrag, Esam E. A. El Sayed, and Ashraf M. T. Elewa. (2020). "ASSESSMENT OF SURFACE WATER RESOURCES FOR IRRIGATION PURPOSES IN ASSIUT GOVERNORATE, UPPER EGYPT.” International Journal of Research - Granthaalayah, 8(3), 156-179. 10.29121granthaalayah.v8.i3.2020.140. 


\section{Introduction}

As a result of increasing population and human activities, pollution became a serious problem affecting the surface and groundwater resources in Assiut Governorate. This study is an expansion of two previous studies carried out on the surface and ground water resources of the western bank of the River Nile between EL-Edwa and Der Mawas cities of Minia Governorate in Upper Egypt (e.g. El Kashouty et al., 2012; Elewa et al., 2013). The main sources of surface and groundwater pollution in this area are the industrial and domestic sewage, and the wastewater. Surface water are mainly exposed to contamination with hazardous industrial waste, fertilizes, and pesticides from agricultural activities, as well as oil pollution brought from ships and oil terminals (EEAAEIMP, 1999 and UN, 2002). Another source of contamination in the study area is the domestic sewage from villages and cities, and human activities. Because the need of water increases due to population growth, industrial development and cultivation of desert land, the availability of water of acceptable quality in Egypt is limited and getting even more restricted. The main purpose of the present study is to assess and to evaluate the quality of both surface and ground water for the irrigation purposes. The most damaging effects of poor-quality irrigation water are excessive accumulation of soluble salts and/or sodium in soil. Highly soluble salts in the soil make soil moisture more difficult for plants to extract, and crops become water stressed even when the soil is moist. When excessive sodium accumulates in the soil, it causes clay and humus particles to float into and plug up large soil pores. This plugging action reduces water movement into and through the soil, thus crop roots do not get enough water even though water may be standing on the soil surface.

\section{Location of The Study Area}

The study area is located on the eastern and western banks of the River Nile between the cities of Dirout at north and EL-Badary of Sedfa at south within Assiut Governorate. The area is bordered from the east and the west by cultivated areas and limestone plateau, approximately between latitudes $260 \mathrm{o} 15^{-}$and $27 \mathrm{o} 10^{-} \mathrm{N}$, and longitudes $30 \mathrm{o} 40^{-}$and $31030^{-} \mathrm{E}$ (Figure 1). Assiut Governorate includes 11 cities, 56 main villages, and 235 subordinate villages, and the number of its population is about 4 million people, form which $73 \%$ are living in the rural area and $27 \%$ are living in the urban area.

\section{Surface Water System}

The surface water hydrology of Assiut governorate is principally represented by the regime of water in the irrigation canals and agricultural drains. Assiut governorate gets its surface water from the River Nile and lateral irrigation canals , EL-Ibrahimia, Naga Hamadi El-Sharqia, Naga Hamadi El-Gharbia canals as a main canals ( first level canals), El-Malah, Beni Ghaleb and other lateral major canals ( second level canals), in addition the distribution canal (third level canals- mosqas) and irrigation ditches (Merwas). Flow in the main canals is continuous while in the branch and distribution canals it is on rotation basis. Also El-Zenar drain represent the main drain in study area in addition the distribution drains (second and third level drain) Figures $2(a, b)$. 


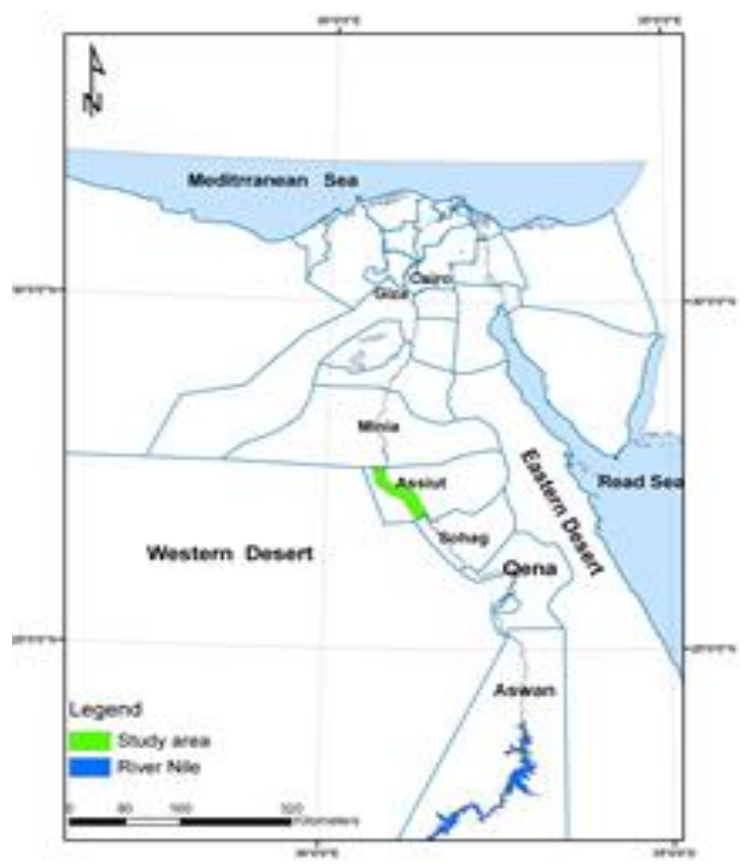

Figure 1: Location map of study area in Assiut Governorate

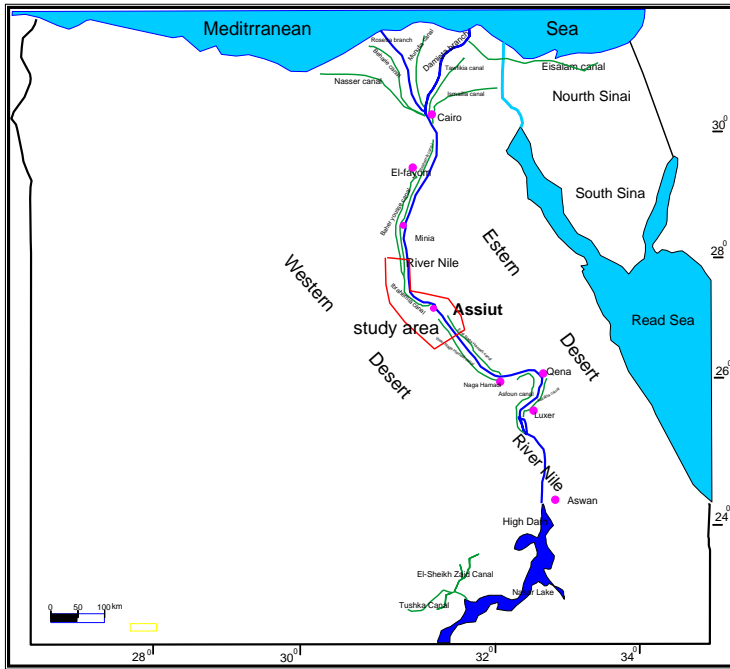

(a)

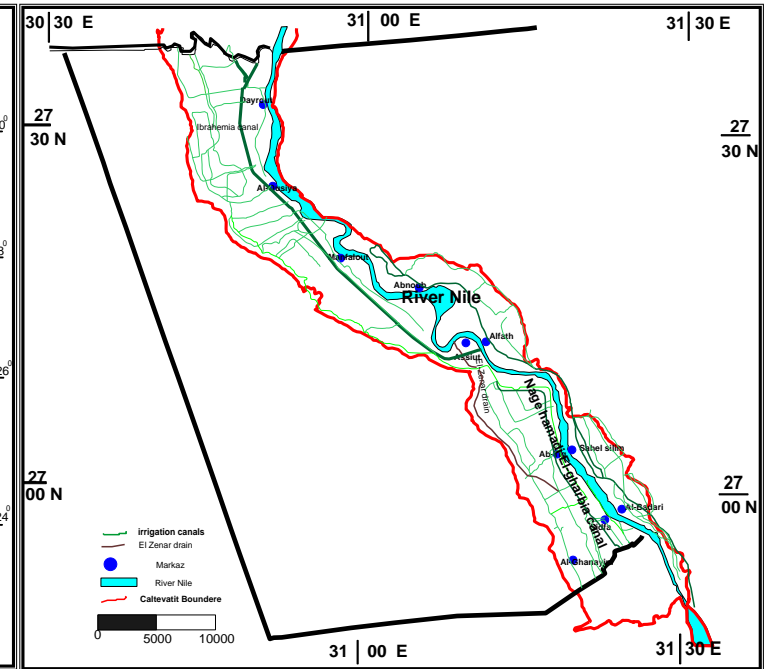

(b)

Figure 2: (a,b) Map of Egyptian with River Nile, main canals and Mine Irrigation canals and their tributaries and El-Zenar drain study area (a-after Shmrukh et al. 2011)

\section{River Nile}

Represent the main sources of fresh water in Assiut governorate. its extend for distances about 160 $\mathrm{km}$ toward the north, the share Assiut governorate from River Nile water about $2.68 \mathrm{BCm} /$ year more than about $90 \%$ (approximately $2.5 \mathrm{BCm}$ ) for agriculture purpose ,1.3\%( approximately $0.035 \mathrm{BCm}$ ) for drinking and domestic purpose after treatment, and the remain for industrial an another purpose. Inter Assiut governorateat El-Badari Markaz path through all governorate Mrkazs accept EL-Ghanaim Markaz while lie at west Sidfa Markaz. EEAA, (2005). 


\section{Ibrahimia Canal (First Level Canales)}

Downstream of the Aswan dam, there are seven barrages to increase the river water level so that it can flow into first level irrigation canals. One of them is the Ibrahimia canal (with length 350 $\mathrm{km}$ ), completed in 1873, the largest artificial canal in the world. It branches off the left bank of the Nile in Assiut figure $3(a, b)$,

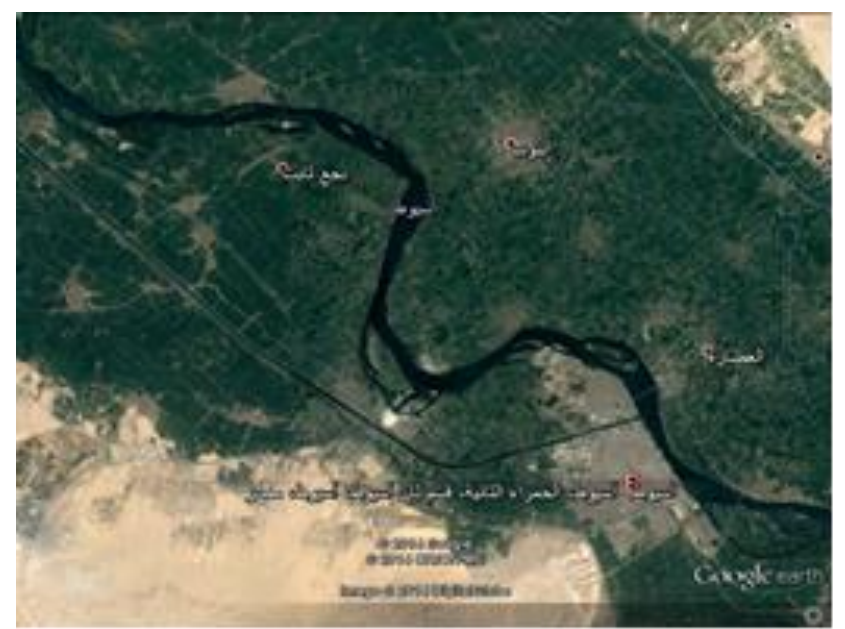

(a)

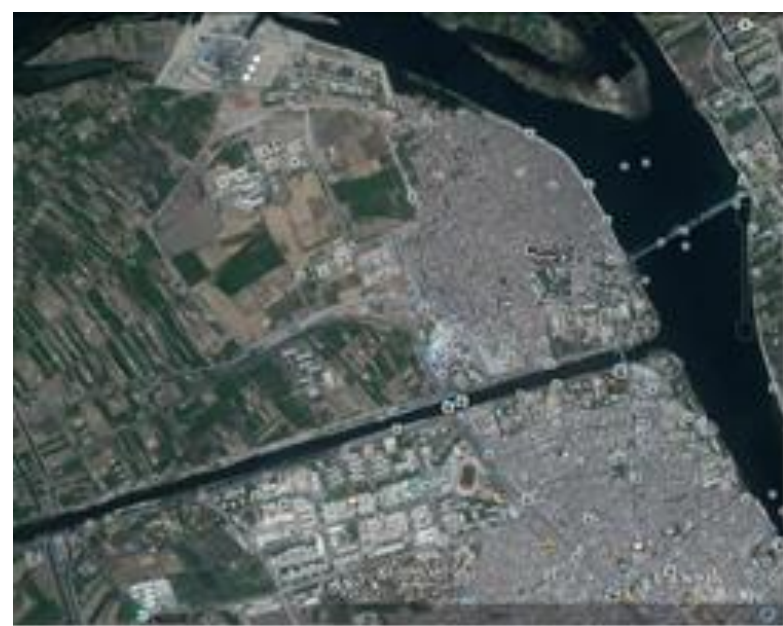

(b)

Figure 3: $(a, b)$ show the outlet of the Ibrahimia canal at Assiut city from River Nile

Whose water level is $50 \mathrm{~m}$ above the sea level, and then runs parallel to the River Nile. Its discharge is increased by the Assiut Barrage completed in 1903. It extend toward the north direction parallel to the river Nile with distance about $55 \mathrm{~km}$ through Assiut governorate figure (1).

\section{Nagi Hamadi El-Sharqia Canal; (First Level Canals)}

Get its water from River Nile at Naga Hamadi Town, at the upstream portion of the old Naga Hamadi barrage where the water level fluctuated between 66.5 to $69 \mathrm{~m}$ above the sea level, and extend toward the north direction parallel to the River Nile, in East portion of Qena and Sohag governorates and inter Assiut governorate (East River Nile) at El-Badari Markaz until riche south El-fathe Markaz figure (4).

\section{Nagi Hamadi El-Gharbia Canal; (First Level Canales)}

Get its water from River Nile at Naga Hamadi Town, at the upstream portion of the old Naga Hamadi barrage where the water level fluctuated between 66.5 to $69 \mathrm{~m}$ above the sea level, and extend toward the north direction parallel to the River Nile, in East portion of Qena and Sohag governorates and inter Assiut governorate (East River Nile) at El-Ghanaim Markaz until riche south Assiut Markaz figure (4). The canals of the irrigation system runfrom south to north nearly parallel to each other as well as to the River Nile. 

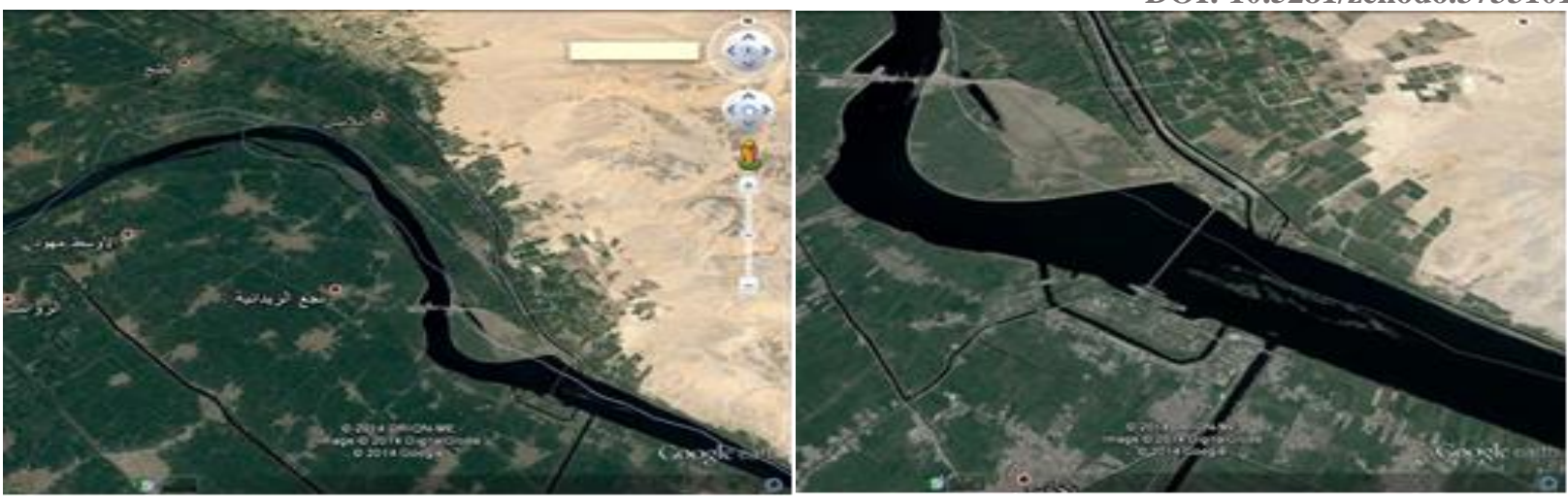

Figure 4: Show the outlet of the Naga Hamadi canals at Naga Hamadi city from River Nile

\section{Drainage Channels}

Drainage channels and drainage through subsurface drains below fields is essential to prevent a deterioration of crop yields from soil salinization. The main drain canal in As suit governorate is EL-Zenar drain, extend toward the west and south west of Assiut city and Markaz as showing in figure (2-b) at the western portion of the River Nile. it receive the most of the waste water (agriculture, domestic and sewage ...ets) from second and third level drain canals which distribution all over the west middle portion of Assiut governorate and from drainage through subsurface drains below fields (direct or indirect), than discharge into the River Nile at Assiut town (downstream portion of Assiut barrage ) figure (2-b).

Surface water system represented the main of recharging source of the groundwater (Quaternary Aquifer) beside seepage from excess irrigation water.

\section{Climate}

The study area is characterized by arid to semiarid, hot climate. The annual average of temperature is about 22.7o (the minimum temperature is $15.3 \mathrm{o}$, and the maximum temperature is about 30.3o). The average rainfall value is about $0.7 \mathrm{~mm} /$ month. The average value of relative humidity is $38 \%$. The average value of evaporation is about $14.2 \mathrm{~mm} / \mathrm{year}$. The winds reach the average of 7.5 Knot/hour (Egyptian metrological Authority,1981-2001) and (EEAA, 2005)

\section{Materials and Methods}

To evaluate the surface water resources in Assiut Governorate for irrigation purposes, the total of 30 surface water samples were collected from irrigated surface water, respectively (Figure 2-b). We used the GPS instrument (Figure 5-a) to locate the studied sites where the water samples were collected. The water analyses were carried out, according to the methods adopted by Rainwater and Thatcher (1950) and those described by Fishman and Friedman (1985), in the Geology Department, Faculty of Science, Minia University, and the Agency of Environmental Affairs of Assiut Governorate. The $\mathrm{pH}$, electrical conductivity, and temperature are measured in situ using Ultra meter tm $6 \mathrm{p}$ (Figure 5-b). The $\mathrm{Cl}, \mathrm{HCO}_{3}, \mathrm{Ca}$, and $\mathrm{Mg}$ elements were measured by titration, while SO4 was estimated by turbidity, and the $\mathrm{Na}$ and $\mathrm{K}$ elements were defined by the flame photometer. The samples were acidified with ultrapure nitric acid, after filtration, to avoid 
complication and adsorption. The acidification was accomplished in situ and in case of toxic metals determination. Then the samples were transported to the laboratory and stored in a refrigerator at approximately $200 \mathrm{C}$ to prevent change in volume due to evaporation. The toxic metals (As, Ba, $\mathrm{Cd}, \mathrm{Cr}, \mathrm{Cu}, \mathrm{Pb}, \mathrm{Ni}, \mathrm{Mn}, \mathrm{Fe}, \mathrm{NO} 2$, and $\mathrm{NH} 4$ ) were determined by the ICP (Inductive Couples Plasma)-AES (Optima 3000; Perkin Elmer). The analyses were carried out at the Agency of Environmental Affairs in Assiut Governorate. The results of laboratory and field measurements were within the limit of $10 \%$, and therefore a significant alteration of the alkalinity during storage and transport can be excluded.

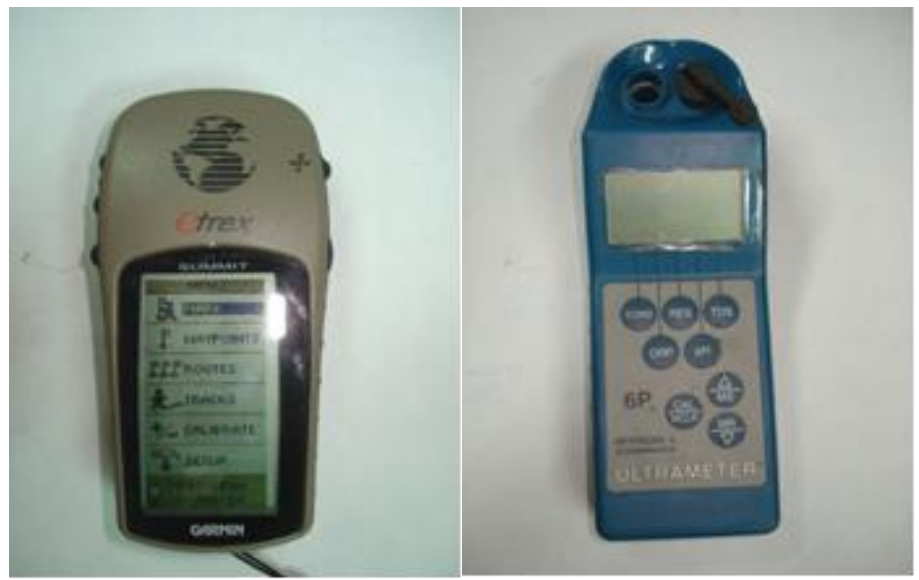

Figure 5: (a, b) Global Positioning system (GPS) and Ultrameter tm 6p

\section{Results and Discussion}

Soil scientists use common categories to describe the irrigation water effect on crop production and soil quality (e.g. salinity hazard, sodium hazard, chloride, boron, nitrate, PH and total alkalinity). The other potential irrigation water contaminants that might affect the suitability of agricultural use are heavy metals and microbial contaminants. Quality standards for irrigation water are based on important factors that affect the productivity of the crops. Several authors have proposed many different classifications for irrigation water. Considering the quality of water and their suitability for irrigation purposes, a number of concepts must be taken into consideration; these are:

1) The total concentration of soluble salt (TDS).

2) The relative proportion of sodium to other cataions (SAR).

3) The residual sodium carbonate (RSC).

4) The concentration of certain minor constituents, especially Boron.

It is notable that the quality requirements of irrigation water vary between crops types and drain ability of soils and climate.

The recommended water quality criteria for irrigation (according to FAO, 1985, 2010) and the guidelines for interpretation of water quality for irrigation (according to Ayers, 1977; Ayers and Wesrcot,1985, Eaton,1950, leeden et al. 1990,) are presented in Tables 3,4,5,6,7,8 and 9. 
DOI: 10.5281/zenodo.3733101

Table 1: Chemical analysis of surface water samples in study area

\begin{tabular}{|c|c|c|c|c|c|c|c|c|c|c|c|c|c|c|c|c|c|c|c|c|c|c|c|c|}
\hline Site No & & PH & TDS & $\mathbf{C a}$ & Mg & $\mathrm{Na}$ & CL & $\mathrm{HCO3}$ & EC & T.Alk & HN4 & NO3 & $\mathrm{Fe}$ & $\mathrm{Cu}$ & Cd & $\mathbf{C r}$ & $\mathbf{P b}$ & Mn & $\mathbf{N i}$ & Zn & B & As & SAR & RSC \\
\hline 1 & \multirow{13}{*}{ 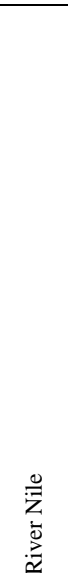 } & 8 & 248 & 34 & 11.2 & 27 & 14 & 115 & 0.292 & 145 & 0.1 & 0.709 & 0.0026 & 0.085 & 0 & 0.0028 & 0.0094 & 0 & 0.056 & 0.091 & 0.02 & 0.035 & 1.027 & -0.235 \\
\hline 2 & & 8.4 & 232 & 39 & 12.8 & 26.8 & 12.7 & 175.3 & 0.311 & 132 & 0.08 & 0.699 & \begin{tabular}{|l|}
0.0024 \\
\end{tabular} & 0.11 & 0 & 0.0032 & 0.0015 & 0.001 & 0.014 & 0.087 & 0.032 & 0.032 & 0.95 & -0.13 \\
\hline 3 & & 8.5 & 234 & 34 & 13.1 & 28.3 & 13 & 121.9 & 0.31 & 135 & 0.2 & 0.824 & \begin{tabular}{|l|}
0.0021 \\
\end{tabular} & 0.096 & 0 & 0.0095 & 0.0084 & 0.01 & 0.01 & 0.047 & 0.051 & 0.018 & 1.045 & -0.779 \\
\hline 4 & & 8.3 & 232 & 34 & 11.9 & 31.1 & 14.1 & 126,21 & 0.307 & 127 & 0.06 & 0.908 & 0.0023 & 0.078 & 0 & 0.0031 & 0.003 & 0.005 & 0.036 & 0.071 & 0.03 & 0.027 & 1.17 & -0.61 \\
\hline 5 & & 8.2 & 233 & 35 & 12.1 & 26.1 & 16.3 & 88.4 & 0.28 & 140 & 0.17 & 0.811 & 0.0025 & 0.09 & 0 & 0.0041 & 0.0046 & 0.02 & 0.031 & 0.039 & 0.023 & 0.012 & 0.97 & -1.259 \\
\hline 6 & & 8.9 & 235 & 37.7 & 14 & 33.4 & 12.5 & 145.8 & 0.339 & 145 & 0.3 & 0.77 & \begin{tabular}{|l|l}
0.0057 \\
\end{tabular} & 0.107 & 0.00015 & 0.0055 & 0.1 & 0.1 & 0.042 & 0.022 & 0.2 & 0.016 & 1.18 & -0.65 \\
\hline 7 & & 8.7 & 229.3 & 33.4 & 13.3 & 25.3 & 14.8 & 90.2 & 0.291 & 136 & 0.14 & 0.56 & \begin{tabular}{|l|}
0.0054 \\
\end{tabular} & 0.095 & 0 & 0.0024 & 0.0099 & 0.13 & 0.009 & 0.073 & 0.25 & 0.024 & 0.93 & -1.29 \\
\hline 8 & & 8.57 & 255.5 & 39.84 & 15.4 & 34.5 & 20 & 201.2 & 0.332 & 152 & 0.011 & 0.822 & 0.0024 & 0.097 & 0 & 0.0045 & 0.0037 & 0.03 & 0.028 & 0.034 & 0.15 & 0.009 & $\begin{array}{l}1.176 \\
\end{array}$ & 0.036 \\
\hline 9 & & 8.8 & 203 & 37.2 & 14.1 & 28.9 & 8 & 160.5 & 0.264 & 122 & 0.08 & 0.46 & 0.0018 & 0.00043 & 0.00006 & 0.00103 & 0.00042 & 0.02 & 0.015 & 0.003 & 0.08 & 0.021 & 1.021 & $\begin{array}{l}-0.39 \\
\end{array}$ \\
\hline 10 & & 7.97 & 533 & 33.8 & 16.1 & 30.4 & 55.5 & 148.8 & 0.711 & 131 & 0.3 & 2.2 & 0.0032 & 0.00016 & 0.0005 & 0.0011 & 0.0039 & 0.01 & 0.097 & 0.0076 & 0.29 & 0.008 & 1.78 & -0.576 \\
\hline 11 & & 8.76 & 189 & 35 & 15.5 & 29.7 & 5 & 200.7 & 0.262 & 130.5 & 0.07 & 0.43 & 0.005 & 0.0022 & 0.00009 & 0.008 & 0.0014 & 0.02 & 0.13 & 0.0061 & 0.35 & 0.075 & 1.047 & 0.235 \\
\hline 12 & & 8.83 & 189 & 38 & 12.8 & 25.8 & 6.5 & 170.4 & 0.258 & 126 & 0.5 & 0.006 & \begin{tabular}{|l|l}
0.0067 \\
\end{tabular} & 0.0011 & 0.00003 & 0.0018 & 0.00088 & 0.007 & 0.085 & 0.0066 & 0.07 & 0.0025 & 0.996 & -0.161 \\
\hline 13 & & 8.83 & 186 & 35 & 11.9 & 25 & 6 & 162 & 0.262 & 128.5 & 0.4 & 0.36 & 0.0048 & 0.00013 & 0.00002 & 0.0011 & 0.0005 & 0.004 & 0.0074 & 0.0066 & 0.09 & 0.009 & 0.93 & -0.1 \\
\hline 14 & \multirow{14}{*}{ 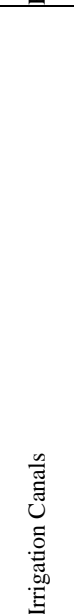 } & 8.9 & 222.8 & 36.6 & 12,5 & 23 & 9 & 147.5 & 0.29 & 133 & 0 & 0.8 & \begin{tabular}{|l|l}
0.0024 \\
\end{tabular} & 0.108 & 0 & 0.0046 & 0.0091 & 0.0087 & 0.008 & 0.075 & 0.02 & 0.0041 & 0.84 & $\begin{array}{c}-0.443 \\
\end{array}$ \\
\hline 15 & & 8.1 & 336.5 & 40.92 & 14.1 & 27 & 8 & 162.5 & 0.53 & 195 & 0.09 & 3 & 3.2 & 0 & 0.007 & 0.0034 & 0 & 0.075 & 0.25 & 0.076 & 0.02 & 0.0065 & 0.9 & -0.539 \\
\hline 16 & & 8.74 & 222.8 & 36.6 & 13.2 & 22,8 & 11.2 & 140.3 & 0.29 & 145 & 0.028 & 0.761 & \begin{tabular}{|l}
0.0022 \\
\end{tabular} & 0.79 & 0 & 0.0023 & 0.0047 & 0.095 & 0.098 & 0.15 & 0.05 & 0.018 & 0.826 & -0.616 \\
\hline 17 & & 8.4 & 222.3 & 32.12 & 11,7 & 21 & 10 & 90 & 0.279 & 123 & 0.004 & 1.03 & \begin{tabular}{|l}
0.0023 \\
\end{tabular} & 0.079 & 0 & 0.0047 & 0.0067 & 0.17 & 0.18 & 0.94 & 0.03 & 0.025 & 0.806 & -1.09 \\
\hline 18 & & 8 & 245 & 32 & 10 & 23,5 & 9 & 45 & 0.35 & 152 & 0.025 & 1.7 & 0.8 & 1.8 & 0.002 & 0.03 & 0.0054 & 0.04 & 0.08 & 1.5 & 0.11 & 0.0084 & 0.93 & -2.535 \\
\hline 19 & & 8 & 223 & 43 & 13 & 25,1 & 8 & $\begin{array}{l}190.3 \\
\end{array}$ & 0.35 & 136 & 0.01 & 0.95 & 2.5 & 1.7 & 0.0003 & 0.02 & 0.0032 & 0.1 & 0.1 & 0.3 & 0.095 & 0.0065 & 0.862 & -0.105 \\
\hline 20 & & 8.5 & 215 & 26 & 12.2 & 32,4 & 7 & 90 & 0.33 & 144 & 0.015 & 0.8 & 3.5 & 1.85 & 0.0006 & 0.04 & 0.0051 & 0.03 & 0.06 & 1.7 & 0.21 & 0.0095 & 1.3 & -0.855 \\
\hline 21 & & 8.5 & 170 & 34 & 11,2 & 26.1 & 11 & 125 & 0.226 & 140 & 0.012 & 0.68 & 0.7 & 0.8 & 0.001 & 0.005 & 0.0031 & 0.028 & 0.07 & 0.21 & 0.065 & 0.011 & 0.99 & -0.572 \\
\hline 22 & & 8.3 & 219 & 31 & 13 & 30.1 & 11.5 & 135.2 & 0.324 & 136 & 0 & 0.8 & 0.87 & 0 & 0 & 0.0004 & 0 & 0.051 & 0.08 & 0.075 & 0.023 & 0.02 & 1.144 & 0.41 \\
\hline 23 & & 6.6 & 224 & 41 & 12,8 & 27.2 & 6.5 & 180.2 & 0.395 & 150 & 0 & 1.4 & 1 & 0 & 0 & 0.0008 & 0 & 0.08 & 0.1 & 0.085 & 0.063 & 0.0085 & 0.818 & -0.158 \\
\hline 24 & & 7 & 240 & 36.6 & 11.9 & 29.2 & 6.8 & 165.4 & 0.263 & 142 & 0.004 & 14.9 & 0.003 & 0.0008 & 0.0008 & 0.0006 & 0.004 & 0.005 & 0.008 & 0.007 & 0.102 & 0.0058 & 1.07 & -0.1 \\
\hline 25 & & 7.9 & 228 & 45.1 & 13.4 & 31 & 2 & 185 & 0.326 & 154 & 0 & 0.9 & 0.9 & 0 & 0 & 0.0004 & 0 & 0.039 & 0.08 & 0.082 & 0.015 & 0.0095 & 1.04 & -0.32 \\
\hline 26 & & 7.41 & 320 & 50 & 17 & 32.5 & 16 & 195.5 & 0.434 & 175 & 0.041 & 2.82 & 2 & 0.007 & 0.03 & 0.031 & 0.075 & 0.7 & 0.095 & 0.329 & 0.08 & 0.0057 & $\begin{array}{l}1.039 \\
\end{array}$ & -0.698 \\
\hline 27 & & 7.07 & 334 & 43 & 12.1 & 28.2 & 12 & 175.8 & 0.513 & 210 & 0.052 & 3.1 & 1.4 & 0.06 & 0.008 & 0.009 & 0.0089 & 0.6 & 0.12 & 0.23 & 0.053 & 0.0045 & 0.983 & -0.264 \\
\hline 28 & \multirow{3}{*}{$\begin{array}{l}\text { El- } \\
\text { Zenar } \\
\text { drain }\end{array}$} & 8.3 & 306.2 & 44.7 & 15 & 30 & 49 & 123 & 0.386 & 145 & 0.1 & 5.83 & 0.023 & 0.115 & 0 & 0.005 & 0.004 & 0.01 & 0.009 & 0.3 & 1.1 & 0.024 & 1 & -1.412 \\
\hline 29 & & 7.9 & 578 & 48.48 & 14.8 & 27.2 & 75.5 & 92.1 & 0.758 & 170 & 0.195 & 2.6 & 0.0011 & 0.098 & 0.0007 & 0.003 & 0.0047 & 0.0097 & 0.0085 & 0.0081 & 1.5 & 0.032 & 0.878 & -2.123 \\
\hline 30 & & 7.86 & 407 & 41 & 16 & 31.3 & 57.2 & 103.5 & 0.537 & 125.6 & 0.2 & 2.06 & 0.7 & 0.1 & 0.002 & 0.0045 & 0.077 & 0.01 & 0.0065 & 0.24 & 1.23 & 0.029 & 1.05 & -1.67 \\
\hline
\end{tabular}


Table 2: Guidelines are based on (FAO) handbook 29 and PACE turf observations (2010)

\begin{tabular}{|c|c|c|c|}
\hline \multirow[t]{2}{*}{ Quality parameter } & \multicolumn{3}{|c|}{ Likelihood of soil problems } \\
\hline & Low & Medium & High \\
\hline $\mathrm{ECw}(\mathrm{ds} / \mathrm{m}, \mathrm{mmhos} / \mathrm{cm})$ & $<0.7$ & $0.7-3.0$ & $>$ \\
\hline TDS(mg/l,ppm) & $<450$ & $450-2000$ & $>$ \\
\hline SAR 0- 3 & $\mathrm{ECW}>0.7$ & ECW 0.7-0.2 & ECW $<0.2$ \\
\hline SAR $3-6$ & $\mathrm{ECW}>1.2$ & ECW 1.2- 0.3 & $\mathrm{ECW}<0.3$ \\
\hline SAR $6-12$ & ECW $>1.9$ & ECW 1.9- 0.5 & ECW $<0.5$ \\
\hline SAR $12-20$ & $\mathrm{ECW}>2.9$ & ECW 2.9- 1.3 & $\mathrm{ECW}<2.9$ \\
\hline Sodium $\mathrm{Na}(\mathrm{me} / \mathrm{l})$ & $<3$ & $3-9$ & $>9$ \\
\hline Sodium Na (mg/l, ppm) & $<70$ & $70-200$ & $>200$ \\
\hline RSC (me/l) & $<1.25$ & & 1.25 \\
\hline Nitrate NO3 -N (mg/l,ppm) & $<5$ & $5-20$ & $>30$ \\
\hline Ammonium NH4-N(mg/l,ppm) & $<5$ & $5-20$ & $>20$ \\
\hline Boron B(mg/l,ppm) & $<0.5$ & $0.5-3.0$ & $>3.0$ \\
\hline Bicarbonate HCO3(me/l) & $<1.5$ & $1.5-8.5$ & $>8.5$ \\
\hline Bicarbonate HCo3 (mg/l,ppm) & 92 & $92-520$ & $>520$ \\
\hline Chloride CL(me/l) & $<3$ & & $>3$ \\
\hline Chloride CL(mg/l,ppm) & $<105$ & & 105 \\
\hline
\end{tabular}

Table 3: Guideline for interpretation of water quality for irrigation (FAO, 1985)

\begin{tabular}{|c|c|c|c|c|}
\hline \multirow{2}{*}{$\begin{array}{l}\text { Potential irrigation water } \\
\text { quality problem }\end{array}$} & \multirow[t]{2}{*}{ Parameter } & \multicolumn{3}{|c|}{ Degree of restriction on use } \\
\hline & & None & Slight to moderate & Severe \\
\hline \multirow{2}{*}{$\begin{array}{l}\text { Salinity (affects crop water } \\
\text { availability) }\end{array}$} & ECiw $(\mathrm{mmho} / \mathrm{cm})$ & $<0.7$ & $0.7-3.0$ & $>3.0$ \\
\hline & Or TDS (mg/l) & $<450$ & $450-2,000$ & $>2,000$ \\
\hline \multirow{6}{*}{$\begin{array}{l}\text { Infiltration } \\
\text { (affects water infiltration rate, } \\
\text { evaluated by using ECiw and } \\
\text { SAR together) }\end{array}$} & SAR & \multicolumn{3}{|c|}{ ECiw (mmho/cm) } \\
\hline & $0-3$ & $>0.7$ & $0.7-0.2$ & $<0.2$ \\
\hline & $3-6$ & $>1.2$ & $1.2-0.3$ & $<0.3$ \\
\hline & $6-12$ & $>1.9$ & $1.9-0.5$ & $<0.5$ \\
\hline & $12-20$ & $>2.9$ & $2.9-1.3$ & $<1.3$ \\
\hline & $20-40$ & $>5.0$ & $5.0-2.9$ & $<2.9$ \\
\hline \multirow{6}{*}{$\begin{array}{l}\text { Specific ion toxicity } \\
(\text { affects sensitive crops) } \\
\left(\mathrm{Na}^{+}\right) \text {surface irrigation } \\
\text { sprinkler irrigation } \\
(\mathrm{Cl}-) \text { surface irrigation } \\
\text { sprinkler irrigation } \\
\text { Boron }\left(\mathrm{B}^{+++}\right)\end{array}$} & SARadj & $<3$ & $3-9$ & $>9$ \\
\hline & $\mathrm{meq} / \mathrm{l}$ & $<3$ & $<3$ & \\
\hline & & & & \\
\hline & $\mathrm{meq} / \mathrm{l}$ & $<4$ & $4-10$ & $>10$ \\
\hline & meq/1 & $<3$ & $<3$ & \\
\hline & $\mathrm{Ppm} / 1$ & $<0.7$ & $0.7-3.0$ & $<3.0$ \\
\hline $\begin{array}{l}\left(\mathrm{HCO}_{3-}\right) \text { Bicarbonate } \\
\text { (overhead sprinkler only) }\end{array}$ & meq/l & $<1.5$ & $1.5-8.5$ & $>8.5$ \\
\hline
\end{tabular}




\begin{tabular}{|c|c|c|c|}
\hline \multicolumn{4}{|c|}{ Plugging potential from irrigation water used in micro irrigation system } \\
\hline Problem & Low & Medium & Severe \\
\hline Physical Suspended Solids (ppm) & $<50$ & $50-100$ & $>100$ \\
\hline Chemical & & & \\
\hline $\mathrm{Ph}$ & $<7.0$ & $7.0-8.0$ & $>8.0$ \\
\hline TDS (ppm) & $<500$ & $500-2000$ & $>2000$ \\
\hline Manganese (ppm) & $<0.1$ & $0.1-1.5$ & $>1.5$ \\
\hline Iron (ppm) & $<0.1$ & $0.1-1.5$ & $>1.5$ \\
\hline Hydrogen sulfide (ppm) & $<0.5$ & $0.5-2.0$ & $>2.0$ \\
\hline Biological Bacteria pop. (no./ml) & $<10,000$ & $10,000-50,000$ & $>50,000$ \\
\hline
\end{tabular}

1 Adapted from Western Fertilizer Handbook, 2002, Ninth edition, California Plant Health Association, Interstate Publications, Inc., Danville, Illinois.

Table 4: Recommended water quality criteria for irrigation (Ayers 1977)

\begin{tabular}{|c|c|c|c|c|c|}
\hline \multirow[t]{2}{*}{ constituents } & \multirow[t]{2}{*}{ Unit } & \multicolumn{3}{|c|}{ Suitability for irrigation } & \multirow[t]{2}{*}{ Specific crops affected } \\
\hline & & Suitable & Marginal & Unsuitable & \\
\hline $\mathrm{EC}$ & $\mu \mathrm{mmhos} / \mathrm{cm}$ & $<750$ & $750-3000$ & $>3000$ & \\
\hline TDS & $\mathrm{mg} / \mathrm{l}$ & $<250$ & $500-2000$ & $>2000$ & \\
\hline $\mathrm{B}^{++}$ & $\mathrm{mg} / \mathrm{l}$ & $<0.5$ & $0.5-2$ & $>2$ & $\begin{array}{l}\text { Fruit and citrius trees } 5-1 \\
\mathrm{mg} / \mathrm{l} \& \text { field crops } 1-2 \mathrm{mg} / \mathrm{l} \\
\& \text { crasses } \mathrm{mg} /\end{array}$ \\
\hline $\mathrm{CL}^{-}$ & $\mathrm{mg} / \mathrm{l}$ & $<142$ & $142-355$ & $>355$ & $\begin{array}{l}\text { Tree crops and ornamentals } \\
\text { - root adsorption Field and } \\
\text { vegetable crops- foliar } \\
\text { damage at }>106 \mathrm{mg} / \mathrm{l}\end{array}$ \\
\hline SAR & & $<3$ & $3-9$ & $>9$ & Tree crops- root adsorption \\
\hline $\mathrm{SO}_{4-}$ & $\mathrm{mg} / \mathrm{l}$ & $<350$ & $350-600$ & $>600$ & \\
\hline
\end{tabular}

Table 5: Guidelines for interpretations of water quality for irrigation (Ayers and wesrcot 1985).

\begin{tabular}{|c|c|c|c|c|c|}
\hline \multirow{3}{*}{$\begin{array}{l}\text { Potential irrigation Properties } \\
\text { Crop Effects } \\
\end{array}$} & \multirow[t]{3}{*}{ Units } & \multicolumn{4}{|c|}{ Degree of restriction on use } \\
\hline & & \multirow[t]{2}{*}{ None } & \multicolumn{2}{|c|}{ Slight to moderate } & \multirow[t]{2}{*}{ Severe } \\
\hline & & & & & \\
\hline Salinity & $\mathrm{EC}_{\mathrm{w}}(\mathrm{ds} / \mathrm{m})$ & $<0.7$ & $0.7-3.0$ & $>3.0$ & \\
\hline \multicolumn{6}{|l|}{ Soil Effects } \\
\hline \multirow{5}{*}{ Infiltration } & $\mathrm{SAR}=0-3$ & \multirow{5}{*}{$\begin{array}{l}\text { And } \\
\mathrm{EC}_{\mathrm{w}}\end{array}$} & $>0.7$ & $0.7-0.2$ & $<0.2$ \\
\hline & $3-6$ & & $>1.2$ & $1.2-0.3$ & $<0.3$ \\
\hline & $6-12$ & & $>1.9$ & $1.9-0.5$ & $<0.5$ \\
\hline & $12-20$ & & $>2.9$ & $2.9-1.3$ & $<1.3$ \\
\hline & $20-40$ & & $>5.0$ & $5.0-2.9$ & $<2.9$ \\
\hline \multicolumn{6}{|l|}{ Crop Effects } \\
\hline \multirow[t]{3}{*}{ Specific ion toxicity } & Sodium SAR & $<3.0$ & $3.0-9.0$ & $>9.0$ & \\
\hline & Chloride(meq/l) & $<4.0$ & $4.0-10.0$ & $>10.0$ & \\
\hline & Boron(mg/l) & $<0.7$ & $0.7-3.0$ & $>3.0$ & \\
\hline
\end{tabular}




\begin{tabular}{|l|l|l|l|c|c|}
\hline \multirow{2}{*}{ Potential irrigation Properties } & \multirow{2}{*}{ Units } & \multicolumn{4}{c|}{ Degree of restriction on use } \\
\cline { 3 - 6 } & & None & Slight to moderate & Severe \\
\hline \multirow{2}{*}{ Miscellaneous } & Nitrogen(mg/l) & $<5$ & $5.0-30$ & $>30$ & \\
\cline { 2 - 6 } & Bicarbonate(meq/l) & $<1.5$ & $1.5-8.5$ & $>8.5$ & \\
\cline { 2 - 6 } & $\mathrm{PH}$ & \multicolumn{5}{|c|}{ Normal range 6.5-8.4 } & \\
\hline
\end{tabular}

Table 6: Eaton classification (1950) based 0n (RSC)

\begin{tabular}{|c|c|}
\hline Suitability of water samples for irrigation & Values of RSC in epm \\
\hline Safe & $<1.25$ \\
\hline Marginal & $1.25-2.5$ \\
\hline Unsuitable & $>2.5$ \\
\hline
\end{tabular}

Table 7: Shown limits of boron in irrigation water (leeden et. Al. 1990) permissible limits boron (in parts per million)

\begin{tabular}{|c|c|c|c|}
\hline \multirow{2}{*}{ Class of water } & \multicolumn{4}{|c|}{ Crop group } \\
\cline { 2 - 5 } & Sensitive & Semi- tolerant & Tolerant \\
\hline Excellent & $<0.33$ & $<0.67$ & $<1.00$ \\
\hline Good & 0.33 to 0.67 & 0.67 to 1.33 & 1.00 to 2.00 \\
\hline Permissible & 0.67 to 1.0 & 1.33 to 2.00 & 2.00 to 3.00 \\
\hline Doubtful & 1.0 to 1.25 & 2.00 to 2.5 & 3.00 to 3.75 \\
\hline Unsuitable & $>1.25$ & $>2.5$ & $>3.75$ \\
\hline
\end{tabular}

The application of these standards to the chemical data in the studied area tables (1) revealed the following results:

\subsection{Salinity and Total Dissolved Solids}

Electrical conductivity (EC) and salinity usually contribute to the total dissolved solids (TDS). The problem occurs when the salts accumulate in the root level in an extent preventing the crop to be able to extract sufficient water from the salty soil solution, causing a water stress for a significant period. If water uptake is appreciably reduced, the plant slows its rate of growth. The most influential water quality guideline on crop productivity is the water salinity hazard that is measured by electrical conductivity (ECw). The primary effect of the high $\mathrm{ECw}$ on crop productivity is the inability of the plant to compete with ions in the soil solution for water. The higher the ECw, the lower the level of water content available to plants; even though the soil may appear wet (Yours et al., 2009). For surface water samples, the TDS values range from 170 to $578 \mathrm{mg} / \mathrm{l}$ within the range of Low Likelihood of soil problems, except at sites number 10 and 29 where TDS values are medium likelihood of soil problems (Figure 6). 


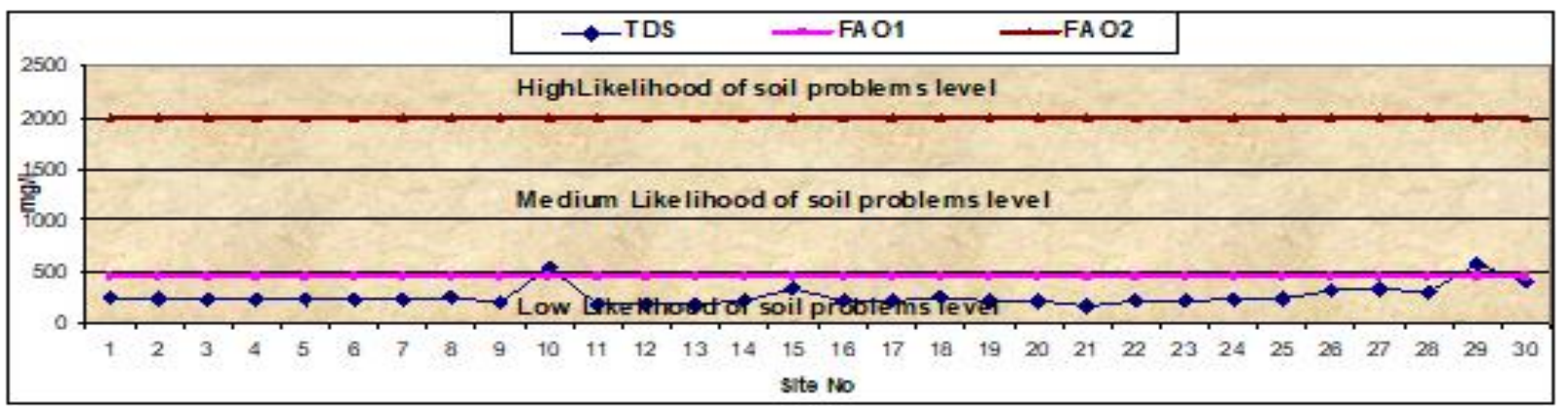

Figure 6: Histogram show classification of surface water samples according TDS hazard (FAO, 19985, 2010)

\subsection{Sodium Hazard}

\subsubsection{Sodium Ions}

Sodium toxicity is often reduced if sufficient calcium is available in the soil. Excessive sodium causes mineral particles of soil to disperse and water penetration to decrease. High sodium concentration accompanied by decrease in the infiltration rate causes problem as the crop could not be adequately supplied with water especially when the hydraulic conductivity of the soil profile is too low to provide adequate drainage. If calcium and magnesium are the predominant adsorbed cations on the soil exchange complex, the soil tends to be easily tilled and have a readily permeable granular structure (Yousry, et al., 2009).

the sodium concentration in surface water samples(Figure 7), ranges between $12 \mathrm{mg} / \mathrm{l}(0.544$ $\mathrm{meq} / \mathrm{l})$ to $34.5 \mathrm{mg} / 1(1.56 \mathrm{meq} / \mathrm{l})$ indicating that there is no restriction of using sensitive crops (Ayers and Westcot, 1985), and lies within the low likelihood of soil problem according to FAO (1985).It is notable that sodium is toxic to certain plants and causes an adverse effect on the soil structure, infiltration, and permeability characteristics (El-Sherbini et al., 1997).

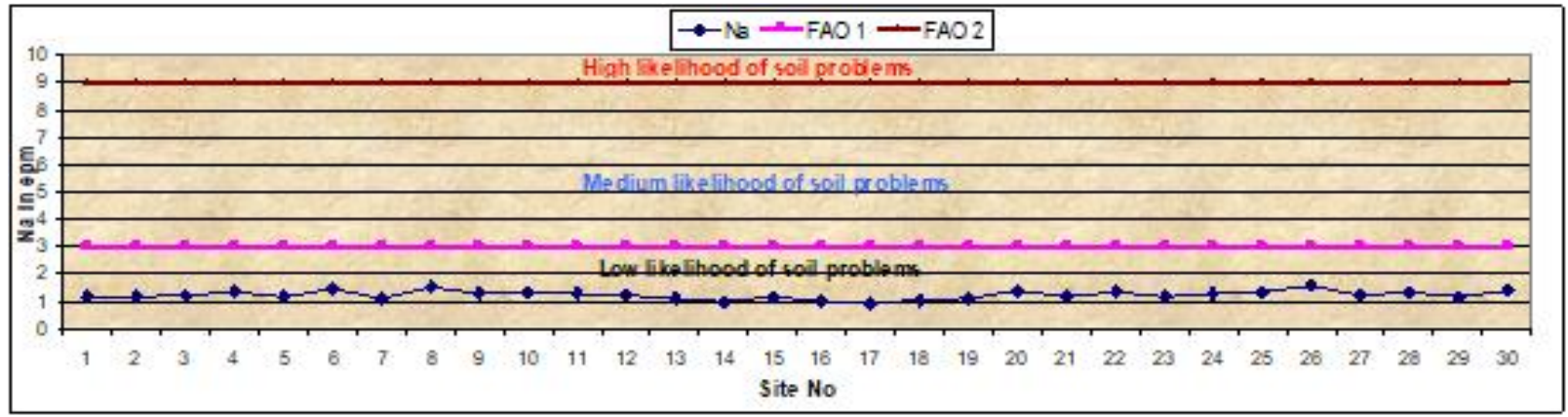

Figure 7: Histogram show classification of surface water samples according $\mathrm{Na}+$ hazard (FAO, 19985, 2010)

Investigations exhibit that the excessive sodium exceeds three times as much as calcium. This high sodium content (>3:1) often results in severe water infiltration problem due to soil dispersion and plugging of the surface pores; this effect is similar to that of water with low salinity. 


\subsection{Infiltration Concerns}

Calcium, magnesium, and sodium are used to calculate the sodium adsorption ratio (SAR) of the irrigation water. The adjusted SAR is calculated using information from (Suarez 1981), which includes the bicarbonate content of the irrigation water. The permeability hazard of an irrigation water sample is related to both the SAR and EC of the irrigation water (Flynnl, 2009).

An infiltration problem occurs if the irrigation water does not enter the soil rapid enough during a normal irrigation cycle to replenish the soil with water needed by the crop before the next irrigation. Low salinity water (less than $0.5 \mathrm{ds} / \mathrm{m}$ and especially below $0.2 \mathrm{ds} / \mathrm{m}$ ) is corrosive and tends to leach surface soils free of soluble minerals and salts, especially calcium, reducing their strong stabilizing influence on soil aggregates and soil structure without salts and without calcium. The soil disperses and the dispersed finer soil particles fill many of the smaller pore spaces, sealing the surface and greatly reducing the rate at which water infiltrates the soil surface. We herein used the method of (Richards 1954) to evaluate the infiltration potential (e.g. the sodium adsorption ratio; SAR).

\subsubsection{Sodium Adsorption Ratio (SAR)}

The sodium adsorption ratio (SAR) is used to estimate the sodality hazard of the Water. SAR is a measure of the tendency of the irrigation water to the soil clay minerals with sodium ions, sodium clays have poor structure and develop permeability problems (George, 1983).

$\mathrm{SAR}$ is defined as in equation

$S A R=\frac{\mathrm{Na}^{+}}{\sqrt{\left(\mathrm{Ca}^{2+}+\mathrm{Mg}^{2+}\right) / 2}}$

Where $\mathrm{Na}^{+}, \mathrm{Ca}^{+}$and $\mathrm{Mg}^{+}$represent meq $1^{-1}$ of sodium, calcium and magnesium ions, respectively. SAR values were evaluated according to FAO guidelines.

According to the U.S. salinity staff classification (Richards 1954) which based on the sodium adsorption ratio (SAR) and the specific conductance (in micro mhos) the water divided into four classes (table 9).

Table 9: U.S. salinity staff classification (Richards 1954) which based on the sodium adsorption ratio (SAR) and the specific conductance (in micro mhos) the water divided into four classes.

\begin{tabular}{|c|c|c|c|}
\hline Conductivity & Quality & Range & Usage \\
\hline C1 & $\begin{array}{c}\text { Low salinity } \\
\text { water }\end{array}$ & $\begin{array}{c}100- \\
250\end{array}$ & $\begin{array}{c}\text { Can be used for irrigation of most crops in most } \\
\text { soils with little likelihood that soil salinity develops }\end{array}$ \\
\hline C2 & $\begin{array}{c}\text { Medium } \\
\text { salinity water }\end{array}$ & $\begin{array}{c}250- \\
750\end{array}$ & $\begin{array}{c}\text { Can be used if a moderate amount of leaching } \\
\text { occurs }\end{array}$ \\
\hline C3 & $\begin{array}{c}\text { High salinity } \\
\text { water }\end{array}$ & $\begin{array}{c}750- \\
2250\end{array}$ & $\begin{array}{c}\text { Cannot be used on soil with restricted Drainage } \\
\text { even with adequate Drainage special management } \\
\text { for salinity control may be required and plants with } \\
\text { good salt tolerant should be selected. }\end{array}$ \\
\hline
\end{tabular}




\begin{tabular}{|c|c|c|c|}
\hline $\mathrm{C} 4$ & $\begin{array}{l}\text { Very High } \\
\text { salinity water }\end{array}$ & $>2250$ & $\begin{array}{l}\text { Is not suitable for irrigation under ordinary } \\
\text { conditions but may be used occasionally under } \\
\text { special conditions as the soils must be permeable, } \\
\text { and Drainge must be adequate, irrigation water } \\
\text { must be applied in excess to provide considerable } \\
\text { leaching. }\end{array}$ \\
\hline SAR & quality & Range & Usage \\
\hline S1 & $\begin{array}{c}\text { Low sodium } \\
\text { water }\end{array}$ & $0-10$ & $\begin{array}{l}\text { Can be used for irrigation of almost all soils with } \\
\text { little changes of the development of harmful levels } \\
\text { of exchangeable sodium. }\end{array}$ \\
\hline S2 & $\begin{array}{c}\text { Medium } \\
\text { sodium water }\end{array}$ & $10-18$ & $\begin{array}{l}\text { Will represents an appreciable sodium hazard in } \\
\text { fine-textured soils having high cation exchange } \\
\text { capacity, especially under low leaching conditions, } \\
\text { unless gypsum is present in the soil. }\end{array}$ \\
\hline S3 & $\begin{array}{l}\text { High sodium } \\
\text { water }\end{array}$ & $\begin{array}{c}18- \\
26\end{array}$ & $\begin{array}{l}\text { May produce harmful levels of exchangeable } \\
\text { sodium in most soils and will require special soil } \\
\text { management, good Drainage, high leaching and } \\
\text { organic matter condition. }\end{array}$ \\
\hline S4 & $\begin{array}{c}\text { Very High } \\
\text { sodium water }\end{array}$ & $\begin{array}{l}26- \\
100\end{array}$ & $\begin{array}{l}\text { Is generally unsatisfactory for irrigation purposes } \\
\text { except at low and perhaps land perhaps medium } \\
\text { salinities. }\end{array}$ \\
\hline
\end{tabular}

For surface water samples (Figure 8), the SAR values range from 0.81 to 1.78 ; indicating good (suitable) for irrigation in all soils as they are located in class [C2-S1] except at site no. 29 (at mixed with sewage from sewage treatment to El-Zenar drain) located in class [C3-S1], the water such classes are good (suitable) under ordinary condition but it can be used under specific condition like adequate drainage and leaching (Ayers and westcot, 1985; FAO, 1985).

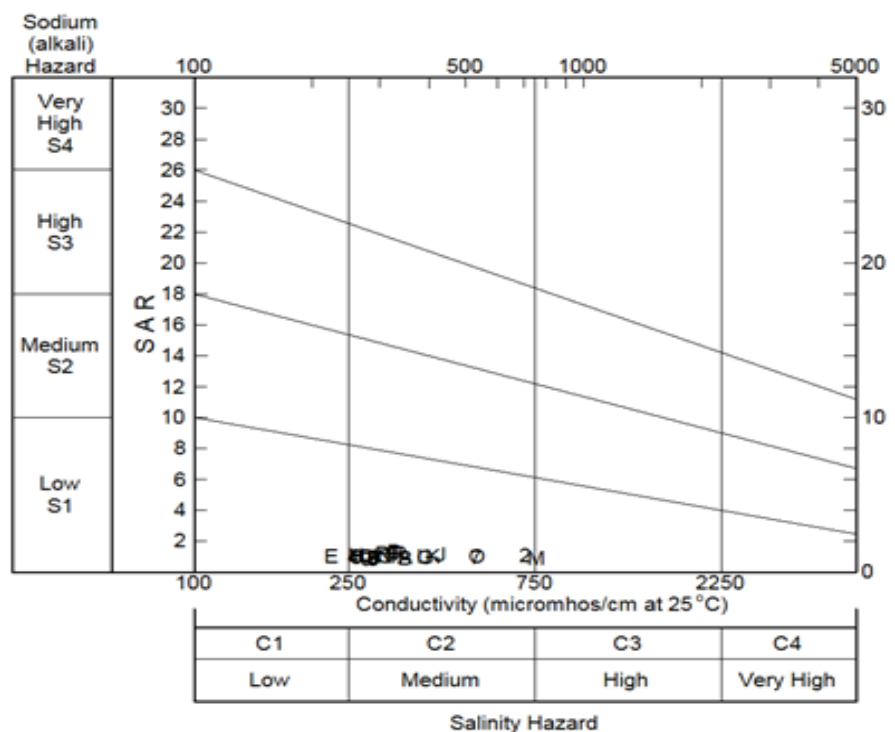

Figure 8: Classification of surface water samples according to U.S. salinity staff classification (Richards 1954) 


\subsubsection{Residual Sodium Carbonates (Eaton's Classification, 1950)}

When the sum of carbonate and bicarbonate is in excess of calcium and magnesium, there is an almost complete precipitation of the latter (Eaton's 1950). This can cause an increase in the proportionate amount of sodium, and so the effect on the soil is the high sodium content. The term residual sodium carbonates (RSC) is defined as follows:

$$
\mathrm{RSC}=\left(\mathrm{CO}^{2+} 3+\mathrm{HCO}_{-3}\right)-\left(\mathrm{Ca}^{2+}+\mathrm{Mg}^{2+}\right) \text { all in mep/L }
$$

The RSC is used to distinguish between the different water classes for irrigation purposes, because the high concentration of bicarbonate leads to an increase in the $\mathrm{PH}$ value, which causes the dissolution of the organic matter. Moreover, the high concentration of the bicarbonate ions in the irrigation water leads to its toxicity and affects the mineral nutrition of plants (see Eaton's classification, 1950).

All the collected surface water samples have RSC values less than 1.25 epm; ranging from -2.535 to 0.4 . They belong to the possibly safe water for irrigation as they are free from residual sodium carbonate (RSC) hazard (Figure 9).

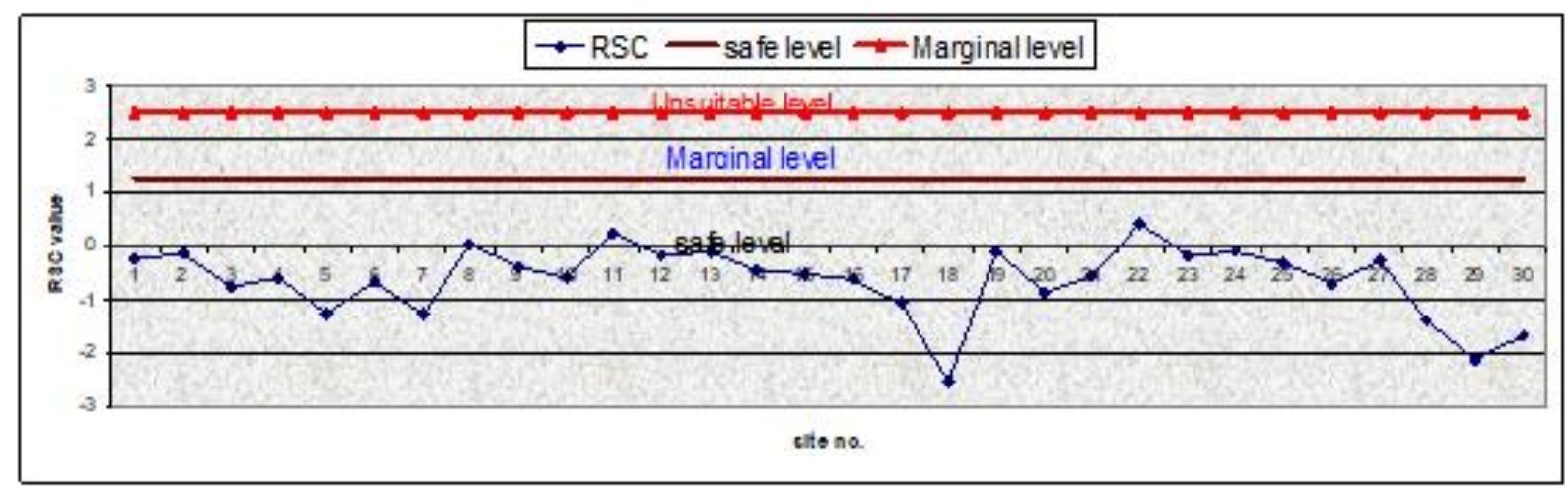

Figure 9: Histogram show classification of surface water samples according RSC values hazard (Eaton 1950)

\subsection{Specific Ion Toxicity}

The specific ion toxicity (like chloride and boron) occurs when the decline of crop growth is due to the excessive concentrations of that specific ion rather than to the osmotic effect alone (Yousry, et al., 2009).

\subsubsection{Chloride}

For surface water samples (Figure 10), chloride ions concentration ranges between $2 \mathrm{mg} / \mathrm{l}(0.056$ meq/l) and $75.5 \mathrm{mg} / \mathrm{l}$ (2.13 meq/l) (Table 3). Comparing the data of chloride with FAO guidelines, it was found that all values are less than $4 \mathrm{meq} / \mathrm{l}$. this means, there is no restriction on using it for some susceptible crops. 
An excess of chloride was detected at the downstream of drains. It is taken as an index of sewage pollution, where sewage water and industrial effluents are rich in chloride, and hence the discharge of these wastes results in high chloride levels in fresh waters (Ravindra et al., 2003).

Most rivers and lakes have chloride concentration lesser than $50 \mathrm{mg} / \mathrm{l}$; therefore, the increase of this value might be an indicative to sewage pollution (Twort et al., 1994).

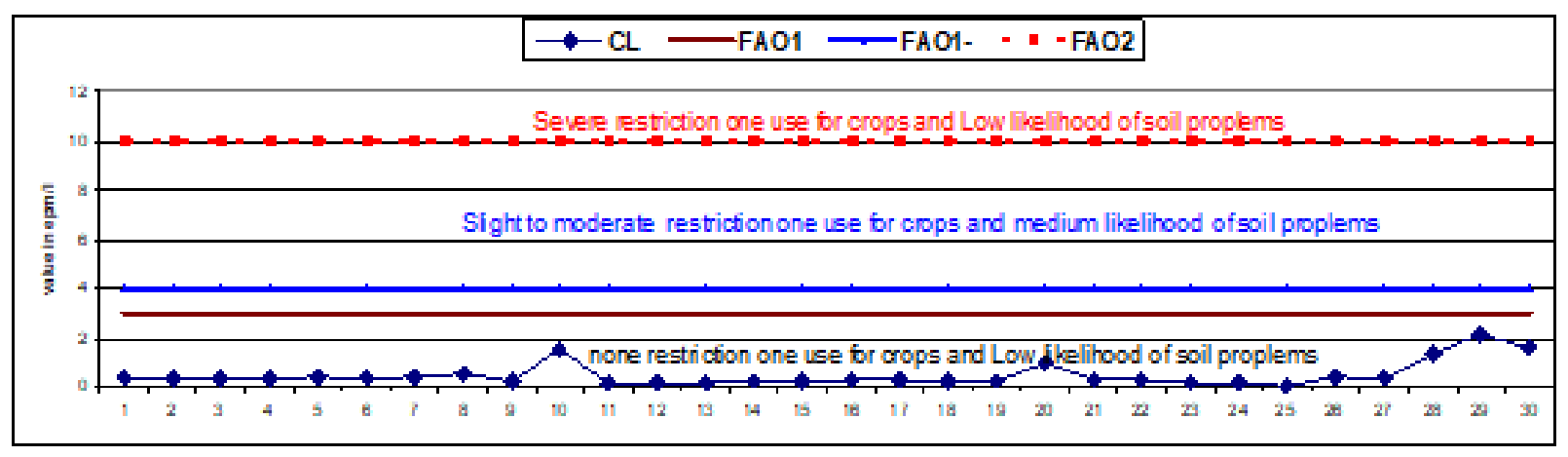

Figure 10: Histogram show classification of surface water samples according Cl- values hazard

\section{Boron}

(FAO, 1985, 2010)

The boron is another element that is essential in low amount for plant growth, but it is toxic at higher concentrations. In fact, toxicity can occur on sensitive crops at concentrations lesser than 1 $\mathrm{mg} / \mathrm{l}$. surface water rarely contain enough boron to be toxic but groundwater occasionally contains toxic amounts. Boron toxicity symptoms normally sow first an older leaf as a yellowing, spotting, or drying of leaf tissue at the tips and edges (Yousry, et al., 2009).

According to (Leaden et al. 1990), the surface and groundwater in the study area can be classified depending on the boron content and the crops tolerant to it in the following manner:

All samples from River Nile and main irrigation canals have B2+ values less than $0.33 \mathrm{mg} / \mathrm{l}$ range between 0.23 and $0.29 \mathrm{mg} / \mathrm{l}$. They belong to the possibly safe water for irrigation of all crops (excellent) except sample No. (11) it's good for Sensitive Crop group and excellent for Semi tolerant and Tolerant Crop group. While samples No 28 and 30 from El-Zenar Drain have B2+ values more than $1 \mathrm{mg} / \mathrm{l}(1.1$ and $1.23 \mathrm{mg} / \mathrm{l})$ respectively, it's doubtful for Sensitive Crop group and good for Semi tolerant and Tolerant Crop group. While sample No 29 from El-Zenar Drain have B2+ values $1.5 \mathrm{mg} / \mathrm{l}$. it's unsuitable for Sensitive Crop group while good for Semi tolerant and good for Tolerant Crop group (Figure 11). 


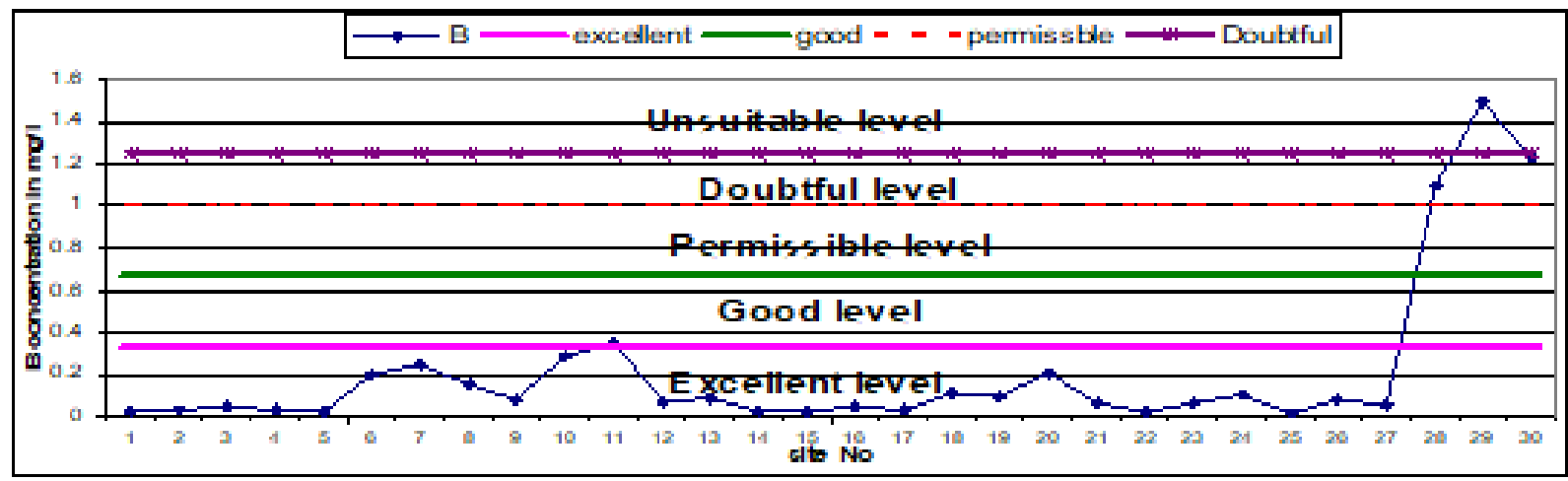

Figure 11: Histogram show classification of surface water samples according B2+concentration on basis of Crop groups of boron tolerance (Leaden, et al., 1990)

While According to (FAO, 1985 \& 2010) guidelines for irrigation water, depended on boron content and likelihood of soil problems can be classified surface and groundwater in study area as the following manner:

all samples from River Nile and main irrigation canals have $B 2^{+}$values less than $0.7 \mathrm{mg} / \mathrm{l}$. (ranged from 0.023 to $0.35 \mathrm{mg} / \mathrm{l}$ ) They belong to low likelihood of soil problems (none degree of restriction on use) for irrigation (the possibly safe water). While samples from El-Zenar drain have B2+ values more than $0.7 \mathrm{mg} / \mathrm{l}$ and less than $3 \mathrm{mg} / \mathrm{l}$.(ranged from 1.1 to $1.5 \mathrm{mg} / \mathrm{l}$ ) They belong to medium likelihood of soil problems (Slight to moderate degree of restriction on use) for irrigation (Figure 12).

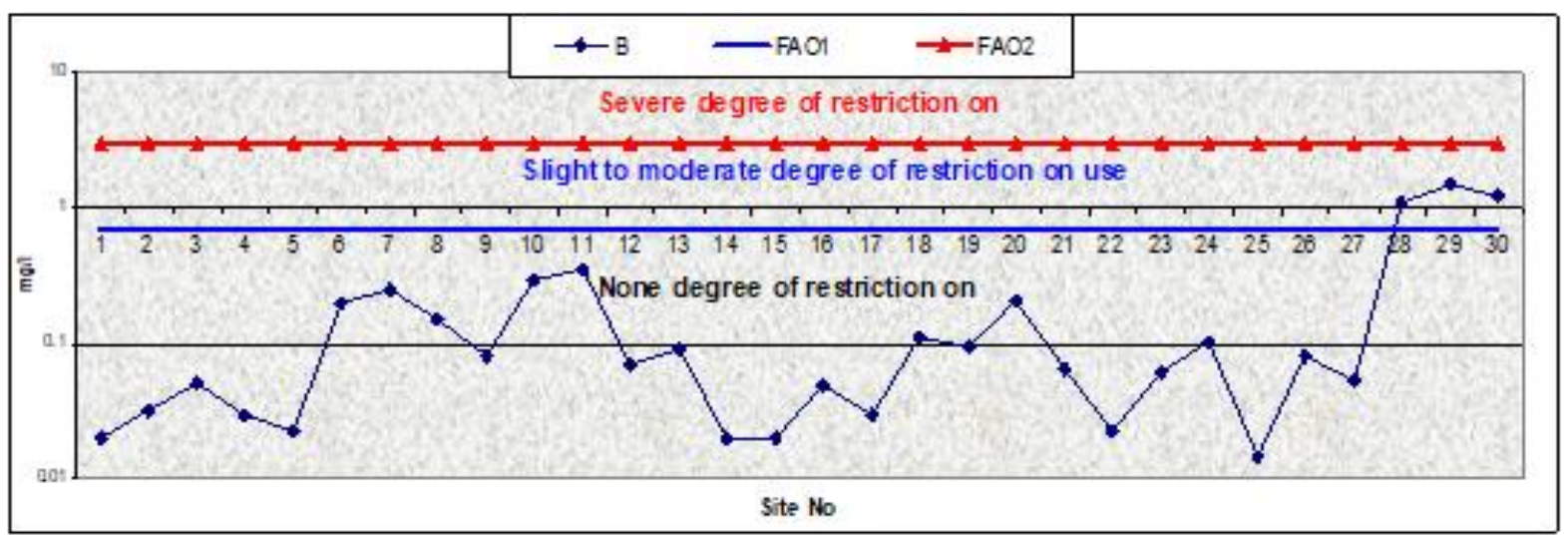

Figure 12: Histogram show classification of surface water samples according B2+ concentration on basis of likelihood of soil problems (FAO, $1985 \& 2010)$

\subsection{Bicarbonates}

The presence of bicarbonate leads to the precipitation of calcium carbonate (scale) at water $\mathrm{pH}$ greater than 7.5. Acidification of the water is the best way to manage bicarbonate, where water levels lesser than $1.5 \mathrm{mg} / \mathrm{l}$ will not cause problems. Severe problems can be noticed at levels above $2.5 \mathrm{mg} / \mathrm{l}$. 
For surface water samples (figure 13), the bicarbonate ions concentration ranges from $45 \mathrm{mg} / \mathrm{l}$ to $201.2 \mathrm{mg} / \mathrm{l}$ (0.74 to $3.3 \mathrm{mg} / \mathrm{l})$, when compared to FAO 1985 guidelines we find that all the surface water samples are considered as belonging to the slightly to moderate restriction on use (medium likelihood of soil problem), except at site no. 18, which shows little problem.

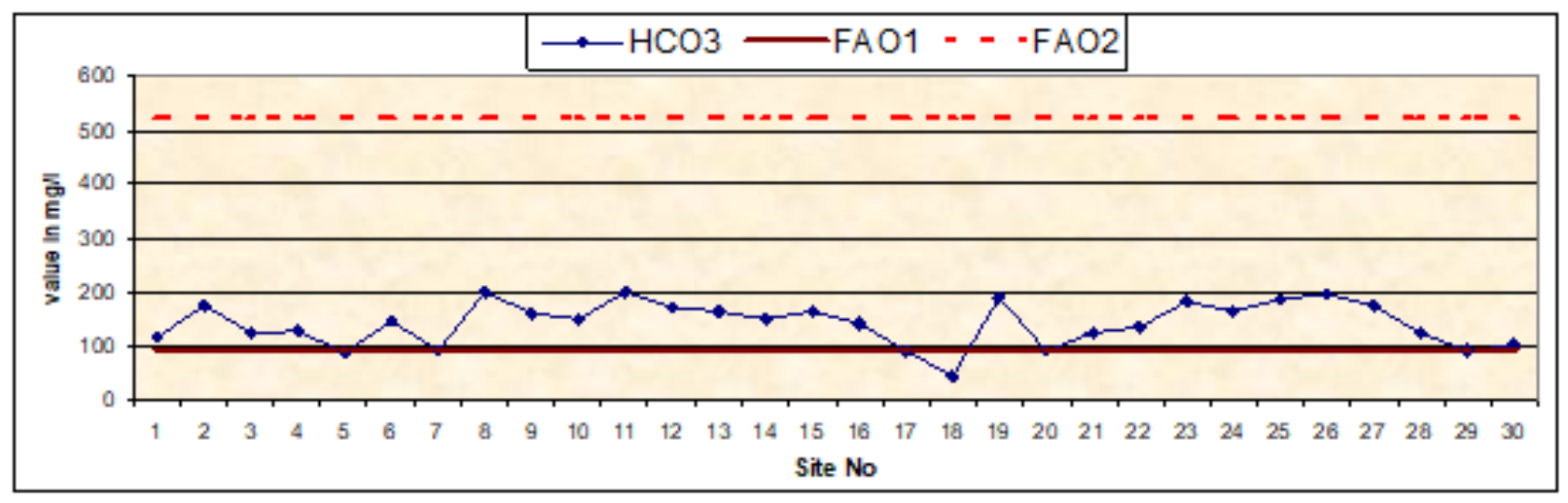

Figure 13: Histogram show classification of surface water samples according HCO3 concentration on basis of likelihood of soil problems (FAO, 1985 \& 2010)

\subsection{Heavy Metals}

Not all trace elements are toxic and many of them are essential for plant growth, but in small quantities (e.g. Fe, Mn, Zn). However, excessive quantities will cause undesirable accumulations in the plant tissues and reduces the growth rate.

By comparing the result with the recommended maximum concentrations of some trace elements in irrigation water (FAO, 1985) and (Rowe et al., 1995) were 5, 5, 2, 0.2, 0.2, 0.2, 0.1, 0.1, 0.05 and 0.01 the maximum limit for lead, iron, zinc, copper, nickel, manganese, arsenic, chromium, cobalt and cadmium, respectively can be obtained the following:.

For surface water, comparing the above recommended values with the data obtained from the analysis of heavy metals revealed that lead, , zinc, copper, arsenic, and chromium values are within the limits recommended by FAO (1985), and ( Rowe et al., 1995) (e.g. none restriction on use). However, the manganese concentration is high at sites no. 26, 27, the cadmium concentration is high at site no 26, the nickel concentration is high at site no. 15, and the iron concentration is high within about $32 \%$ of the studied sites, and therefore lies within the slightly - medium to severe restriction on use.

The Iron concentration ranges between 0.0011 and $3.5 \mathrm{mg} / \mathrm{l}$, the manganese concentration ranges between zero and $0.7 \mathrm{mg} / \mathrm{l}$, the copper concentration ranges between zero and $1.85 \mathrm{mg} / \mathrm{l}$, the recorded cadmium concentration ranges between zero and $0.03 \mathrm{mg} / \mathrm{l}$, the chromium concentration ranges between 0.0004 and $0.04 \mathrm{mg} / \mathrm{l}$, the Lead concentration ranges between zero and $0.1 \mathrm{mg} / \mathrm{l}$, the nickel concentration ranges between 0.0065 and $0.25 \mathrm{mg} / \mathrm{l}$, the zinc concentration ranges between 0.003 and $1.7 \mathrm{mg} / \mathrm{l}$, and the recorded arsenic concentration is less than $0.1 \mathrm{mg} / \mathrm{l}$ in all sites (figure 14). 


\subsection{Miscellaneous Elements}

The miscellaneous elements include the $\mathrm{pH}$ values, the total alkalinity, and the nutrients (ammonia and nitrates)

\subsection{PH Values}

For surface water samples, the $\mathrm{pH}$ values range between 6.6 and 8.9

The increase of $\mathrm{pH}$ values at sites no. 6, 7, 9, 11, 12,13 and 16 could be related to photosynthesis and growth of aquatic plants (Allem, et al., 1969; El-Wakeel et al.,1970). Photosynthesis consumes carbon dioxide leading to the rise of $\mathrm{pH}$ value.

However, chemical reactions in water that are controlled by the $\mathrm{pH}$ values and the biological activity is usually restricted to a fairly $\mathrm{pH}$ range of 5 to 8 (Tebbutt, 1998).

In general, unpolluted streams normally show a near neutral or slightly alkaline. Finally, the $\mathrm{pH}$ values were found to be within the permissible limits of FAO (1985) at sites no. 6, 7, 9, 11, 12, 13 and 16.

The greatest direct hazard of an abnormal $\mathrm{pH}$ value is its impact on the irrigation equipments. The $\mathrm{pH}$ value that is lesser than 6.5 will generally lead to potential corrosion of the irrigation equipments (figure 15).

\section{Total Alkalinity}

For surface water samples, the total alkalinity ranges between 122 to $210 \mathrm{mg} / \mathrm{l}$ about (1.99 to 3.44 meq/l) Alkalinity serves as a $\mathrm{pH}$ reservoir for inorganic carbon. It is usually taken as an index of productive potential of water (Ravindra et al., 2003).

Alkaline water leads to high bicarbonate. The bicarbonate concentrations of surface water ranges between 0.74 and $3.29 \mathrm{meq} / \mathrm{l}$ indicating that there is a slight to moderate restriction of use; however, site no. 8 is considered as belonging to the level of no restriction of use (figure 13). 

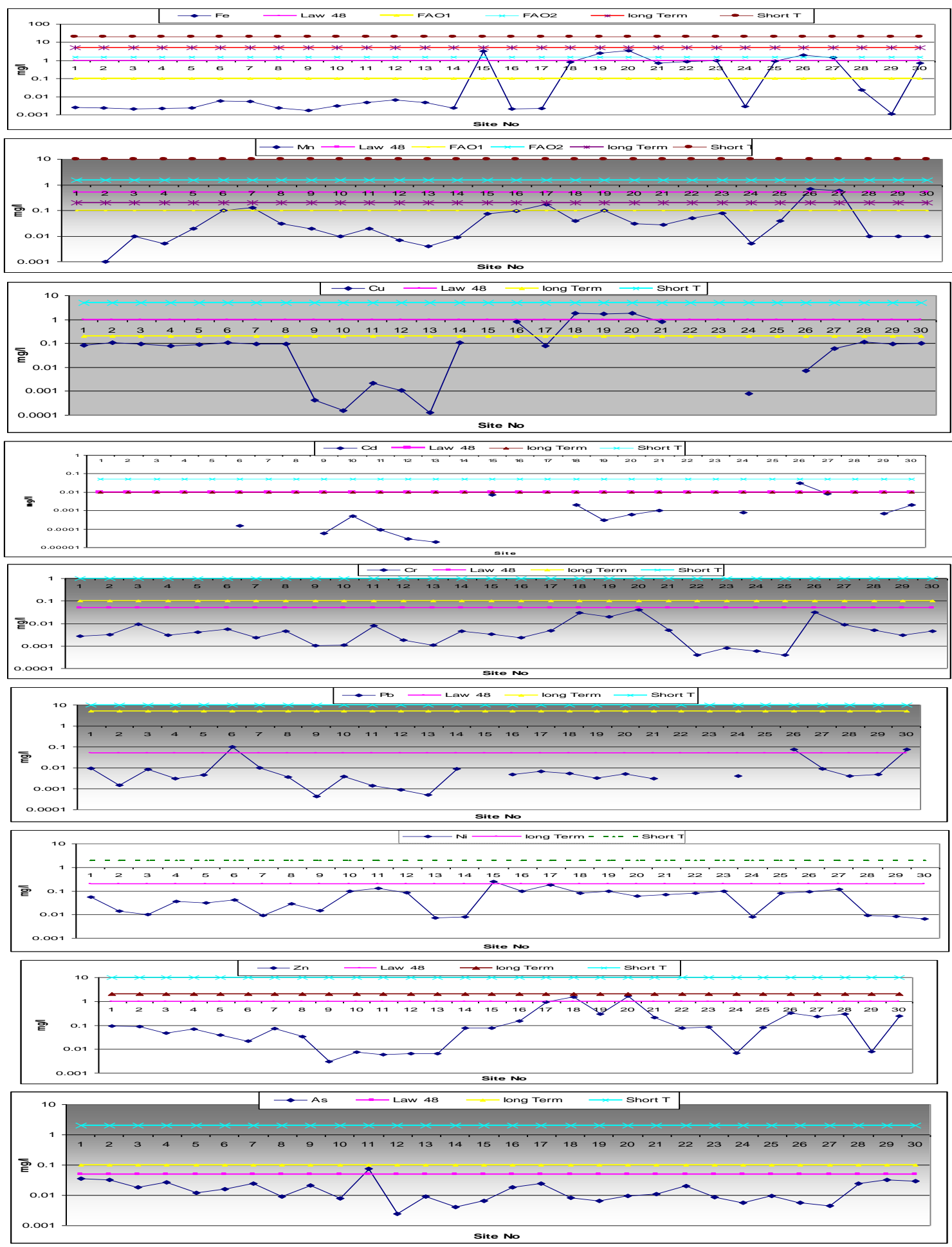

Figure 14: Variation in trace and heavy metals concentration of surface water samples according to recommended limits of (FAO, 1985) guideline for irrigation water and (Rowe, D.R. el at 1995) 


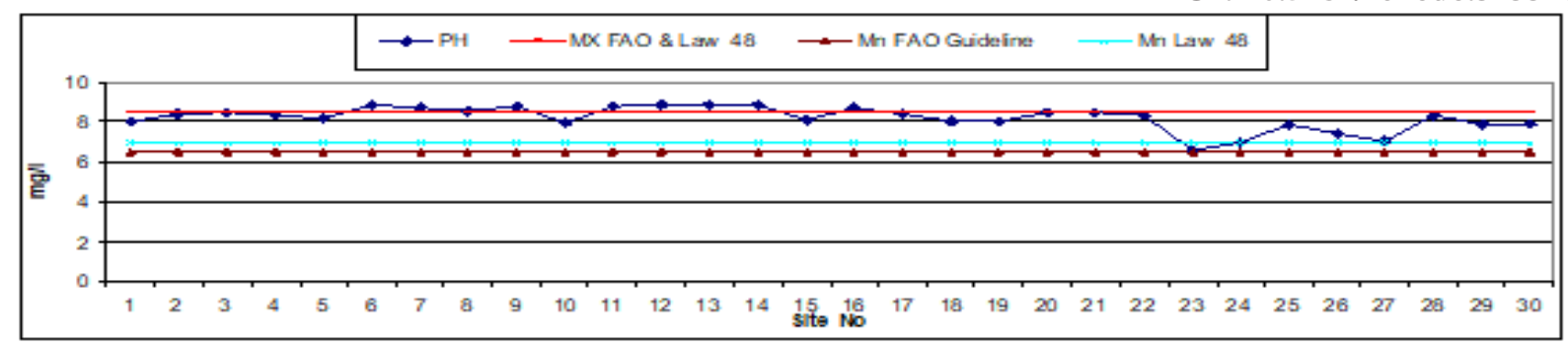

Figure 15: Variation in PH of surface water samples according to recommended limits of (FAO, 1985) guideline for irrigation water

\subsection{Nutrients (Ammonia and Nitrates)}

For surface water samples, the concentration of ammonia (Figure 16) ranges from zero to $0.5 \mathrm{mg} / \mathrm{l}$ within the permissible low level of likelihood of soil problems (none restriction of use at all sites for all crops)

The nitrate concentration within the surface water samples (Figure17) ranges from 0.006 to 14.9 $\mathrm{mg} / \mathrm{l}$ (e.g. within the permissible low level of likelihood of soil problems; none restriction of use at all sites for all crops except the site no. 24, which lies within the level of the slight to moderate restriction of use).

The high concentration of ammonia in wastewater discharging from drain or sewage waste led to the high contamination of the water by ammonia. The ammonia concentration in unpolluted water is lesser than $0.2 \mathrm{mg} / \mathrm{l}$ as nitrogen (chapman, 1992).

By comparing the ammonia and nitrate values with the FAO guidelines $(5 \mathrm{mg} / \mathrm{l} \mathrm{N})$ it is found that there is no restriction on using the studied water samples for sensitive crops except above mentioned sites.

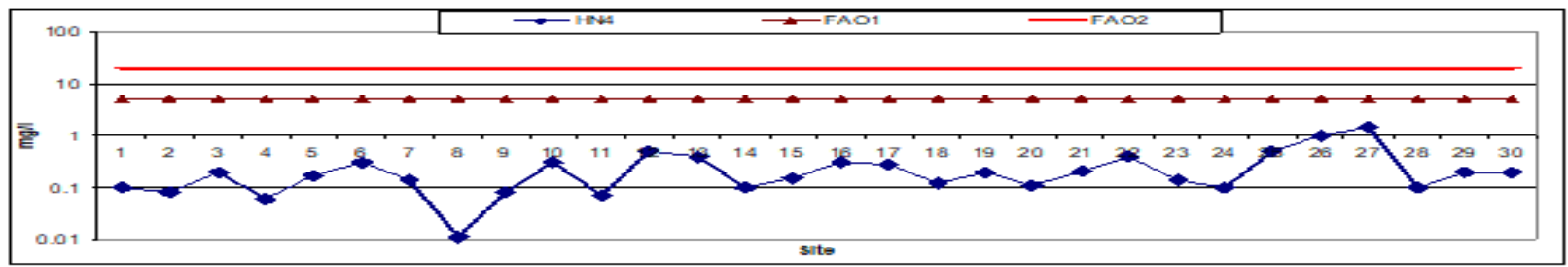

Figure 16: Variation in NH4 concentration of surface water samples according to recommended limits of (FAO, 1985) guideline for irrigation water

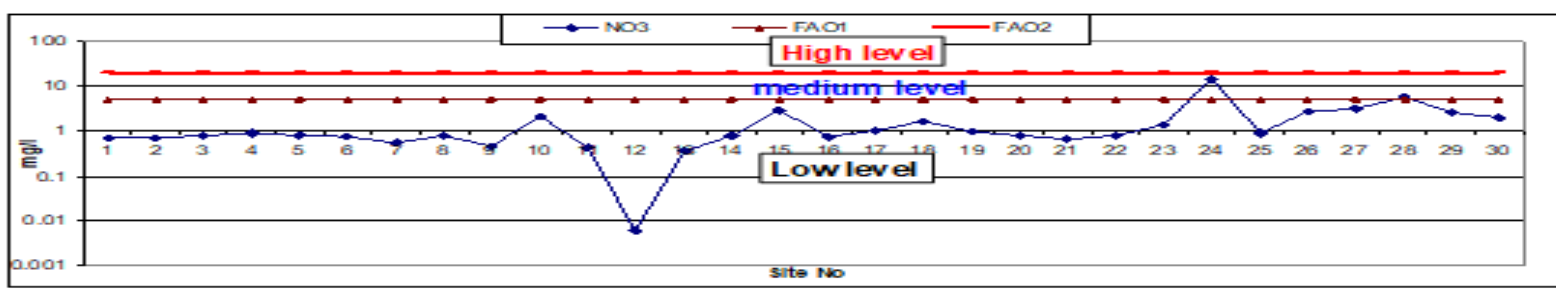

Figure 17: Variation in NO3 concentration of surface water samples according to recommended limits of (FAO, 1985) guideline for irrigation water 


\section{Conclusions and Recommendations}

Our analyses detected the variation in the properties of both the surface and ground waters. All the $\mathrm{PH}$ values of surface water samples are within the recommended limits of FAO (1985), except at sites no. 6, 7, 9, 11, 12,13, 14 and 16, which exhibit more than the recommended limits. All the TDS concentrations in surface water samples are within the recommended limits of FAO (1985) indicating none restoration on use, except at sites no. 10 and 29; these are considered as belonging to the slight to moderate restoration on use. All the Na concentrations at surface water samples are within the recommended limits of FAO (1985) (e.g. none restoration on use). All the Boron concentrations at surface water samples belong to the safe limits of irrigation water for the sensitive crops (excellent), except $16 \%$ of surface water samples which are good for irrigation of Sensitive crop group and excellent for irrigation of semi- tolerant and tolerant crops. There are respectively about $10 \%$ of surface water samples have $\mathrm{B}^{2+}$ values more than $1 \mathrm{ppm}$ (excellent to good for irrigation of tolerant crop group), and good for irrigation of semi-tolerant crop group, while permissible for irrigation of sensitive crop group. Chloride concentrations at surface dwater samples (within the recommended limits). The bicarbonate concentrations in surface water samples are within the moderate -medium level of likelihood of soil problem, except at site no. 18 , which is belonging to the low level of likelihood of soil problem. The NH3 concentrations in all surface water samples are within the recommended limits (none restoration on use) for all sensitive crops, while the NO3 concentrations in all surface and groundwater samples are within the recommended limits (none restoration on use) for all sensitive crops, except at site no. 24, which are within the moderate -medium level of likelihood of soil problem,

Table 8: Shown crop groups of boron tolerance (Leeden et.at.1990)

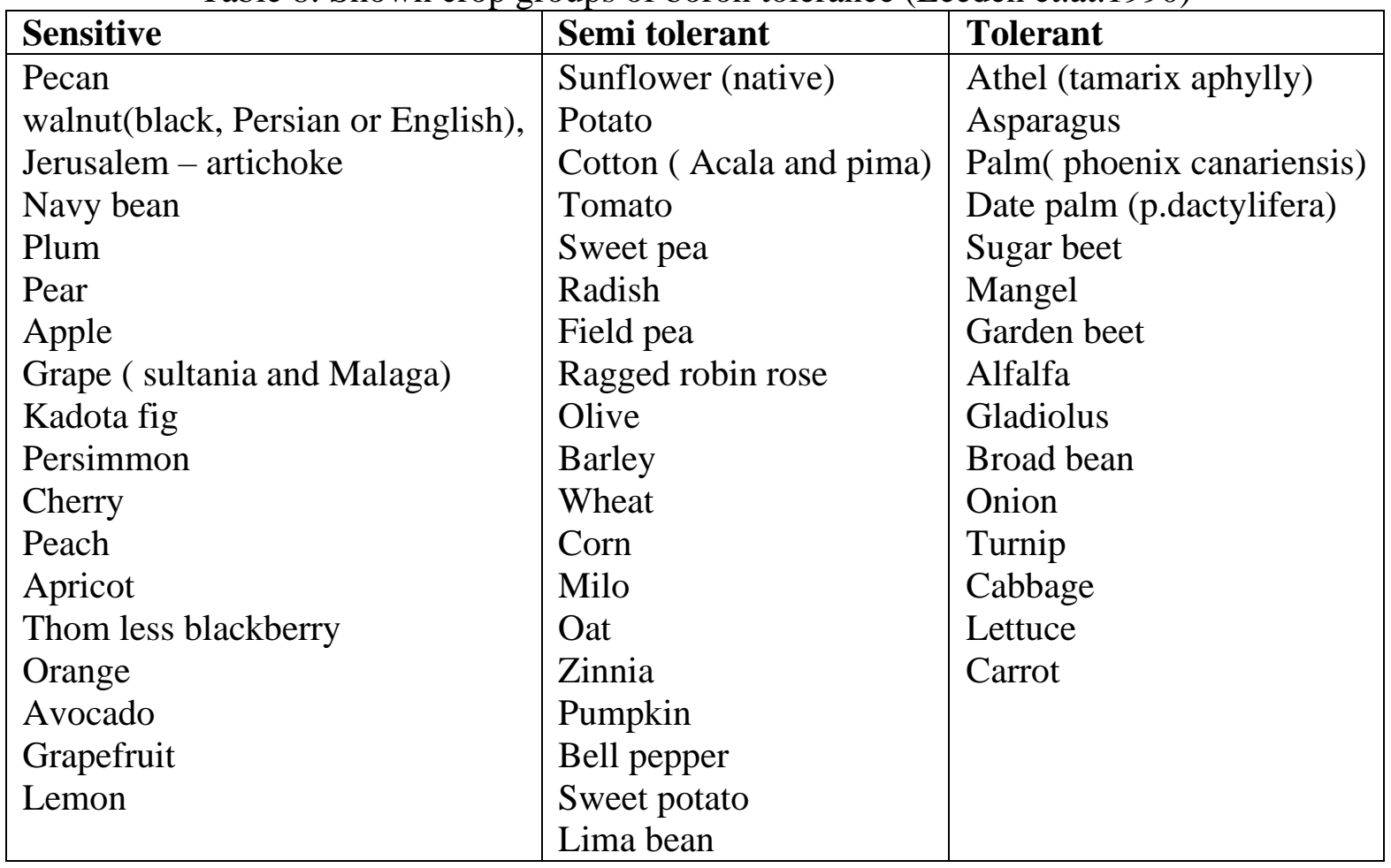


Consequently, we recommend a strong control is needed concerning the use of fertilizers and pesticides in the agriculture purposes, as well as selecting the suitable kind of crops for each area as in (figures 18 and 8) which show classified the surface water in study area based on the hazard of TDS, Na, EC and SAR values and their effected on planet growth and its products in related to soil problems, also as show in details in (figures 19 and 11) and (table 8) which obtained more details based on Boron hazard in related to the sensitivity and tolerant of deferent crops to it. Preventing the direct disposal of sewage, domestic wastewater, and industrial wastewater before treatment to the irrigation canals and the River Nile. We also stress on preventing the construction of open septic tanks, especially near the pumping well.

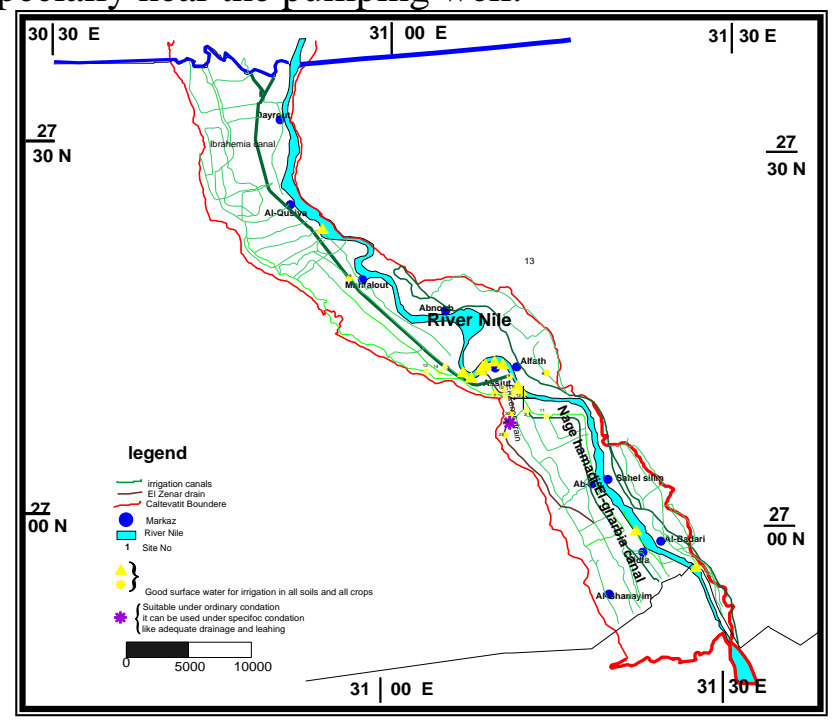

Figure 18: Suitability of surface water for irrigation purpose in related to likelihood soil problems according to recommended limits of (FAO, 1985, 2010) guideline for irrigation water

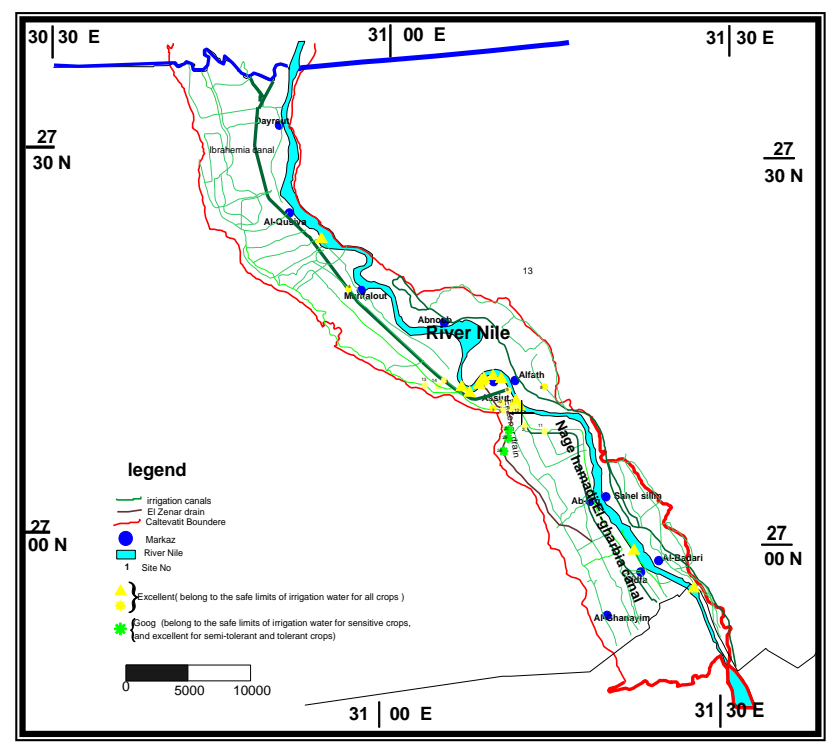

Figure 19: Suitability of Surface water for irrigation purpose in related to Boron hazard and crop groups tolerance according to recommended limits of (FAO, 1985, 2010) and (Leeden et. Al., 1990) guideline for irrigation water 


\section{References}

[1] Allem, A.A. and Samman, A.A. (1969): Productivity of lake Mariut, Egypt part I. physical and chemical aspects, intern. Revue Ges. Hydrobiol., 54, 313 - 355.

[2] Ayers, R. S. and westcot (1985): water quality for agriculture. Food and Agricultural Organization of the United Nations (FAO) irrigation and Drainage Paper 29 Rev. 1,Rome, Italy.

[3] Ayers, R. S., (1977): Quality of water for irrigation "Jour. Of the irrigation and drainage division ASCE, vol. 103. No. IR2.

[4] Chapman, D. (1992): Water quality assessments: A Guide to the Use of Biota, Sediments and Water in environmental Monitoring. Chapman \& Hall, London, (on behalf of UNESCO/ WHO/UNEP). Pp., 585.

[5] Eaton, F.M. (1950): Significance of carbonates in irrigation water. Soil science 39:123-133.

[6] EEAA (2005): Environmental profile of Assiut governorate, (2005)

[7] EEAA-EIMP (1999): Annual report for monitoring of Mediterranean Coastal water for year 1998, Egyptian Environmental Affair Agency.

[8] Egyptian meteorological Authority Egyptian meteorological authority (1981-2001): annual meteorological reports, Cairo.

[9] El Kashoute M., A.,El-Sayed,E.E., Elewa A. M.t., Morsi M. S., (2011): Environmental impact of anthropogenic activities on surface and groundwater systems in the western part of the River Nile, In Minia Governorate, journal of Amirican science , 2012; 8(5) 150 - 161 pp.

[10] Elewa A. M. T., El Kashoute M., A., El-Sayed,E.E., Morsi M. S., (2013): Quantitative study of surface and groundwater systems in the western part of the River Nile, Minia Governorate, Upper Egypt: Water quality in relation to anthropogenic activities, greener journal of physical sciences ISSN: 2276-7851.

[11] El-Sherbini, A., El Fiky, A., El Moattassem, M., Abdel Gawad, s. and Nour El Din, M. (1997): Desigh of monitoring network for water management in the River Nile, Nile 2002, Feb. 1997 , addis ABABA, Ethiopia.

[12] El-Sherbini, A.M., Bary, M.A.A., Heikal, M.T. and Hamdy, A. (1997): Environmental impacts of pollution sources on Rosetta Branch water quality Volume II. Water quality and pollution control. Water management, salinity and pollution control towards sustainable irrigation in the Mediterranean region. CIHEAM international conference, valenzano, Bari, Italy, 22-26 September, 65-85.

[13] El-Wakeel, S. K. and wahby, S. D. (1970): Hydrography and chemistry of lake Mansalah, Egypt. Arch. Hydrobiol., $67,173-200$.

[14] FAO (1985): Food and agriculture Organization of the united nations ( FAO , 1985) irrigation and Drainag " Bull. NO 29 Rev. I, 1985.

[15] Fishman, M. G. and Friedman, L. C. (1985): Methods for determination of inorganic substances in water and fluvial sediments". U. S. Geol. Surv., Book 5, Chapter. A1. Open File Report, pp. 85495, Denver, Colorado, U. S. A.

[16] Flynnl R.(2009): Irrigation Water Analysis and Interpretation,1Extension Agronomist, Agricultural Science Center at Artesia, New Mexico State University Guide W-102.Sc Cooperative Extension Service - College of Agricultural, Consumer and Environmental Sciencesiences This publication is scheduled to be updated and reissued 12/14.

[17] George, P. R. (1983): Agricultural water quality criteria irrigation aspects. Dep. of Agriculture Western Australia, Resource Management Technical Report No. 30.

[18] Leeden, V. F., Troise, F. L. and Todd,D. K.( 1990): the water Encyclopedia". Lewis publishers, 808 p. 
[19] Morsi M. S., (2012): Environmental impact of anthropogenic activities on surface and groundwater systems in the western part of the River Nile, In Minia Governorate' . Msc. 2012 fucl. Sci. geol. Dep. Minia University.MSc.

[20] Rainwater, F. H. and Thatcher, L. L. (1960): Methods for collection and analysis of water samples". U. S. Geol. Surv. Water Supply, paper No. 1454, U. S. A., 301p.

[21] Ravindra, K.; Meenakshi, A.; Rani, M. and kaushik, A. (2003): Seasonal variations in physicochemical characteristics of River Yamuna in Haryana and its ecological best-designated use. Environ. Monit., 5: 419-426.

[22] Richards, L.A. (1954): In USDA Agricultural Handbook" US Department of agriculture, Washington DC, pp., 160.

[23] Rowe,D.R. el at 1995 Rowe, D. R. and Abdel Magid, I. M. (1995): Handbook of Waste-water Reclamation and Reuse". CRC press. Inc. 550p.

[24] Shamrukh M (1999): Effect of chemical fertilizers on groundwater quality in Upper Egypt. PhD thesis, Department of Civil Engineering, Minia University, Egyp

[25] Tebbutt, T. (1998). Principles of water quality control,5th ed., Hallam university

[26] Twort, A.C.; Law, F.M.; Crowley, F.W. and Ratnayaka, D.D. (1994): Water supply. 4th edition, John Wiley and sons, inc., $605 \mathrm{p}$ third A venue . New york, 10158.

[27] UN (2002): Johannesburg Summit 2002, Country profile, Egypt http: //www.un.org /esa/agenda21/natlinfo/ wssd/Egypt.pdf.

[28] Yousry, M., El-Sherbini,A., Heikal, M.,T., Salem,T.,A.(2009): Suitability of water Quality Atatus of Rosetta Branch for west Delta water conservation and irrigation rehabitation project. Water science. Scientific Journal of the National water Research center. Issue, 46.October 2009

*Corresponding author.

E-mail address: geo_mamdoh@yahoo.com 